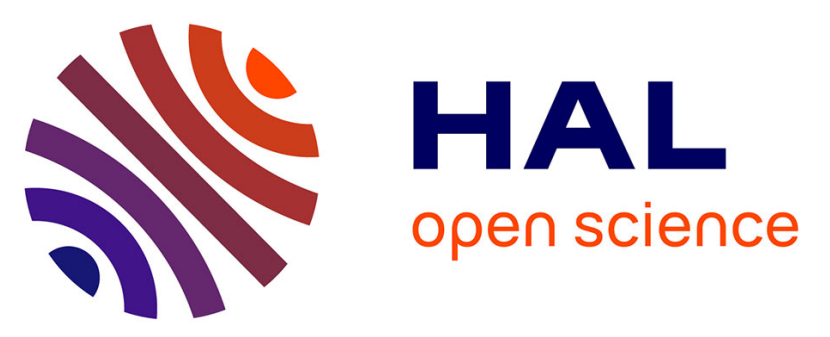

\title{
Study of damped vibrations of a vibroacoustic interior problem with viscoelastic sandwich structure using a High Order Newton solver
}

\author{
B. Claude, L. Duigou, G. Girault, J.M. Cadou
}

\section{To cite this version:}

B. Claude, L. Duigou, G. Girault, J.M. Cadou. Study of damped vibrations of a vibroacoustic interior problem with viscoelastic sandwich structure using a High Order Newton solver. Journal of Sound and Vibration, 2019, 462, pp.114947 -. 10.1016/j.jsv.2019.114947 . hal-03487203

\section{HAL Id: hal-03487203 \\ https://hal.science/hal-03487203}

Submitted on 21 Dec 2021

HAL is a multi-disciplinary open access archive for the deposit and dissemination of scientific research documents, whether they are published or not. The documents may come from teaching and research institutions in France or abroad, or from public or private research centers.
L'archive ouverte pluridisciplinaire HAL, est destinée au dépôt et à la diffusion de documents scientifiques de niveau recherche, publiés ou non, émanant des établissements d'enseignement et de recherche français ou étrangers, des laboratoires publics ou privés.

\section{(c) (1) $\$$}

Distributed under a Creative Commons Attribution - NonCommerciall 4.0 International 


\title{
Study of damped vibrations of a vibroacoustic interior problem with viscoelastic sandwich structure using a High Order Newton solver
}

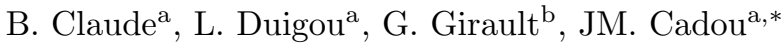 \\ ${ }^{a}$ Univ. Bretagne Sud, UMR CNRS 6027, IRDL, F-56100 Lorient, France \\ ${ }^{b}$ Centre de recherche des Écoles de Saint-Cyr Coëtquidan, Écoles de Coëtquidan, 56381 Guer cedex, France
}

\begin{abstract}
The aim of this study is to compute damped eigenfrequencies and modes of a vibroacoustic interior problem with fluid-structure coupling. Damping is introduced using a sandwich structure with viscoelastic core. In this paper, the coupled problem is solved by a High Order Newton (HON) solver, based on homotopy and perturbation techniques. Thus, the initial non-linear problem is turned into a set of linear algebraic systems, easier to solve. Comparison of the results obtained by the HON solver with those obtained by the Newton classical method highlights the efficiency of the proposed method. Finally, the mechanical behaviour of the coupled damped problem is studied.
\end{abstract}

Keywords: Perturbation method, Vibration, Fluid-structure interaction, Viscoelasticity

\section{Introduction}

Noise and vibration control is a common objective in the industrial field. Whether it is a question of comfort or safety, the fields of application are numerous and varied: transport, building, civil and military engineering... That is why damping solutions are studied. By dissipating vibrational energy, these solutions 5 reduce or cancel vibration and noise. Usually, they are separated into two different categories: one is passive damping and the other one is active damping. Passive damping solutions are based on the use of damping or absorbing materials such as fibrous materials (many bio-based materials [1]: biohemp, wool, wood...), polymer foam or composite materials. Active damping consists in introducing materials or structures that adapt to the vibrations to be damped. It could be for example piezoelectric material, shape-memory alloys (SMA) [2] or smart structures made of microelectromechanical systems (MEMS) [3]. In this study, the chosen solution is a passive one: a polymer material with a viscoelastic behavior integrated into a sandwich structure.

In this paper, a vibroacoustic interior problem with fluid-structure coupling is studied. The free vibrations of a cavity filled with fluid (air or water) are considered. In order to introduce damping, the walls of the cavity are made of a viscoelastic sandwich structure. The viscoelastic behavior is modelled with a complex Young's modulus and two models are examined: the hysteretic model and the generalized Maxwell model. A pressure-displacement formulation is chosen to describe the coupled system. Because of this choice, the matrix system to be resolved is non-symmetric. Moreover, because of the pulsation dependence of the viscoelastic material behavior, the problem is non-linear. Finally, the problem can have a lot of degrees of freedom (complex values, fine spatial discretization needed...).

Various methods can be used to solve such a problem [4] [5] [6]. One of the simplest method is to study the response of the forced vibration problem [7] [8]. Angular frequencies are then estimated by reading the resonance curve. However, this method requires a lot of computational time and does not

\footnotetext{
* Corresponding author

Email address: jean-marc.cadou@univ-ubs.fr (JM. Cadou)
} 
provide very accurate results. Inverse iteration method [6] [9], based on Newton algorithm, computes the 25 smallest angular frequency. The next frequency can be obtained with a shifting or an orthogonalization technique. This method is likely to be costly in computational time because it requires a matrix inversion for each iteration. In the case of low damped problems, modal strain energy (MSE) [10] [11] can be considered. The idea is to compute a damped pulsation and structural damping using real undamped modes or approximation of complex modes. However, results of a non-linear problem are difficult to obtain with MSE. So, if the viscoelastic material behavior depends on the angular frequency, this method must be adapted [12]. Currently, preferred methods to solve non linear problems are based on subspace techniques. By projecting the problem on a subspace chosen for its interesting properties, a smaller matrix system, easier to solve, is obtained. For example, non-linear Arnoldi method [13] uses Krylov subspace and nonlinear Jacobi-Davidson method [14] is based on Rayleigh-Ritz process. Then, eigenvalues and eigenmodes 5 are computed using inverse iteration method or Newton algorithm.

In this study, the considered problem is both non-linear and non-symmetric. Thus, due to these complexities, the previous methods could be very costly in terms of computational time. In order to solve the vibroacoustic problem with reasonable computational time, a High Order Newton (HON) solver based on homotopy and perturbation techniques is proposed in this paper. Initially proposed by Mallil et al. [15], 40 this method has given interesting results to solve viscoelastic sandwich structure vibration problems [16] [17]. This work consists in adapting this technique to vibroacoustic interior problems since it has never been applied to fluid-structure systems. Taking into account the fluid-structure interaction causes numerical difficulties. Indeed, coupling terms appear in the matrices, making it non-symmetrical and poorly conditioned. Thus, the resolution requires powerful algorithms. That's why the most efficient solver of [17], the High Order Newton solver associated with Padé representation, has been chosen for this study.

The outline of the paper is the following: First, the eigenvalue problem to be solved is introduced. Then, Newton's method is reminded and the proposed numerical method is described. Finally, the numerical results are presented in order to validate the proposed method and study the damping influence on the behavior of the viscoelastic cavity.

\section{Governing equations}

In this paper, the free vibrations of a fluid contained in a sandwich cavity are studied. The fluid is inviscid, compressible, non-weighting and fills the whole cavity. In order to reduce vibrations, damping is introduced by using a sandwich structure made of two external elastic layers with a viscoelastic core. The viscoelastic material is modelled with a complex Young's modulus $E(\omega)$.

Several formulations can be considered to describe the fluid-structure interaction problem [18]: displacementdisplacement, pressure-displacement, displacement potential-displacement, velocity potential-displacement... In this study, the chosen formulation is pressure $p$ for the fluid and displacement $\mathbf{u}$ for the solid.

Let $\partial \Omega_{S F}$ be the fluid-structure interface, with $\mathbf{n}_{F}$ the unit normal vector pointing the fluid domain $\Omega_{F}$ outwards. The unit normal vector $\mathbf{n}_{S}$ pointing the solid domain $\Omega_{S}$ outwards is the exact opposite : $\mathbf{n}_{\mathbf{S}}=-\mathbf{n}_{\mathbf{F}}$. With the previous hypothesis, the local dynamic equilibrium equations are:

$$
\begin{aligned}
& \operatorname{div}\left(\boldsymbol{\sigma}_{\boldsymbol{c}}\right)+\omega^{2} \rho_{S} \mathbf{u}=\mathbf{0} \quad \text { in } \Omega_{S} \\
& \boldsymbol{\sigma}_{\boldsymbol{c}} \mathbf{n}_{\mathbf{S}}=p \mathbf{n}_{F} \text { on } \partial \Omega_{S F} \\
& \Delta p+\frac{\omega^{2}}{c^{2}} p=0 \quad \text { in } \Omega_{F} \\
& \boldsymbol{\nabla}(p) \cdot \mathbf{n}_{\mathbf{F}}=\omega^{2} \rho_{F} \mathbf{u} \cdot \mathbf{n}_{\mathbf{F}} \quad \text { on } \partial \Omega_{S F}
\end{aligned}
$$

where scalars $\omega, c, \rho_{F}$ and $\rho_{S}$ are the angular frequency, the sound velocity, the fluid density and the solid density respectively.

Equations (1a) and (1c) represent the solid and the fluid equilibrium respectively. Coupling between fluid and solid domains is introduced by the relations (1b), taking the stress continuity at the interface into account, and (1d), representing the normal velocity continuity at the interface. 
By using the finite element method [19][20][9], the discrete form of the previous equations is obtained:

$$
\left(\left[\begin{array}{cc}
\mathbf{K}_{\mathbf{S}} & -\mathbf{C} \\
0 & \mathbf{K}_{\mathbf{F}}
\end{array}\right]-\omega^{2}\left[\begin{array}{cc}
\mathbf{M}_{\mathbf{S}} & 0 \\
\mathbf{C}^{t} & \mathbf{M}_{\mathbf{F}}
\end{array}\right]\right)\left\{\begin{array}{l}
\mathbf{U}_{\mathbf{n}} \\
\mathbf{P}_{\mathbf{n}}
\end{array}\right\}=\left\{\begin{array}{l}
0 \\
0
\end{array}\right\}
$$

where subscripts $S$ and $F$ stand for the solid and the fluid domains. Vectors $\mathbf{U}_{\mathbf{n}}$ and $\mathbf{P}_{\mathbf{n}}$ are the nodal unknowns for displacement and pressure. The fluid mass matrix $\mathbf{M}_{\mathbf{F}}$, the solid mass matrix $\mathbf{M}_{\mathbf{S}}$ and the

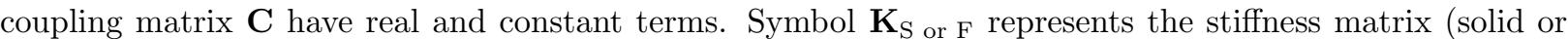
fluid domain). The stiffness matrix of the solid domain $\mathbf{K}_{\mathbf{S}}$ is made of an elastic part $\mathbf{K}_{\mathbf{S}}(\mathbf{0})$ and a viscoelastic part $\mathbf{K}_{\mathbf{S}}(\omega)$ such as $\mathbf{K}_{\mathbf{S}}=\mathbf{K}_{\mathbf{S}}(\mathbf{0})+\mathbf{K}_{\mathbf{S}}(\omega)$. The elastic matrix is constant and real, while the viscoelastic matrix is complex and depends on the angular frequency. This dependence is described in section 3.2.

The previous coupled matrix system (2) can be written as the following generalized eigenvalue problem:

$$
\left[\mathbf{K}(\mathbf{0})+E(\omega) \mathbf{K}-\omega^{2} \mathbf{M}\right] \mathbf{U}=0 \text { with } \mathbf{U}=\left\{\begin{array}{l}
\mathbf{U}_{\mathbf{n}} \\
\mathbf{P}_{\mathbf{n}}
\end{array}\right\}
$$

where the nodal unknowns of displacement $\mathbf{U}_{\mathbf{n}}$ and pressure $\mathbf{P}_{\mathbf{n}}$ are contained in the mixed unknown vector

Due to Young's modulus dependence on the angular frequency, the generalized eigenvalue problem (3) is non-linear. Moreover, viscoelasticity is modelled by a complex Young's modulus, so unknowns $\mathbf{U}, \omega$ and the viscoelastic stiffness matrix $E(\omega) \mathbf{K}$ are complex values, doubling the size of the matrix system (two degrees of freedom correspond to each unknown : a real one and an imaginary one). The global stiffness matrix of the solid elastic and fluid domains $\mathbf{K}(\mathbf{0})$ and the global mass matrix $\mathbf{M}$ have real terms. All the global matrices are non-symmetric due to the presence of the coupling term C. Global matrices are also poorly conditioned because of the difference in order of magnitude between solid and fluid terms. In order to avoid problems caused by poor conditioning, a preconditioning step has been added, as proposed in [21].

In order to simplify the resolution, the eigenvalue problem (3) is rewritten as follows:

$$
[\mathbf{K}(\mathbf{0})+E(\omega) \mathbf{K}+\Omega \mathbf{M}] \mathbf{U}=0 \quad \text { with } \Omega=-\omega^{2}
$$

A classical method to solve the problem (4) is the Newton's method [22] [23]. However, Newton's iterative method is limited by the tangent operator. Indeed, this operator can be poorly conditioned, so the solution gained lacks accuracy. Moreover, convergence is not always obtained, or the algorithm can converge towards a mode different from the expected mode. Finally, the resolution can be costly in CPU time because a matrix factorization is required at each iteration. It is the reason why a high order Newton solver is proposed in 85 the next section.

\section{Proposed method: High Order Newton solver}

\subsection{High Order Newton algorithm}

The HON solver is inspired by a method based on homotopy and perturbation techniques, initially proposed by Mallil et al.[15]. Contrary to Newton's method, based on a first-order development, the HON solver calculates a high order correction using a polynomial expansion. This method has already been used to study vibrations of viscoelastic beams [17] or geometrical and material defaults in beams [26]. In this paper, we propose to apply this method to a vibroacoustic interior problem.

First, unknowns are searched in incremental form:

$$
\begin{aligned}
\mathbf{U} & =\mathbf{U}_{0}+\Delta \mathbf{U} \\
\Omega & =\Omega_{0}+\Delta \Omega \\
E(\omega) & =E_{0}+\Delta E \quad \text { with } E_{0}=E\left(\omega_{0}\right)
\end{aligned}
$$

where the initial point $\left(\mathbf{U}_{0}, \Omega_{0}, E_{0}\right)$ is chosen as the solution of the undamped problem associated to the damped problem (3), as defined in [17]. 
Then, introducing (5) in (4), the problem is written on a quadratic form as proposed in [15]:

$$
\mathbf{L}_{\mathbf{t}}\left(\mathbf{X}_{0}\right) \boldsymbol{\Delta} \mathbf{X}+\mathbf{L}\left(\mathbf{X}_{0}\right) \boldsymbol{\Delta} \mathbf{X}+\mathbf{Q}(\boldsymbol{\Delta} \mathbf{X}, \boldsymbol{\Delta} \mathbf{X})=-\mathbf{R}_{0}
$$

95

here the following terms are identified:

- Unknown vector $\Delta \mathrm{X}$ :

$$
\Delta \mathbf{X}=\left\{\begin{array}{lll}
\Delta \mathbf{U} & \Delta \Omega & \Delta E
\end{array}\right\}^{t}
$$

- Unknown vector at initial point $\mathbf{X}_{0}$ :

$$
\mathbf{X}_{0}=\left\{\begin{array}{lll}
\mathbf{U}_{0} & \Omega_{0} & E_{0}
\end{array}\right\}^{t}
$$

- Initial residual vector $\mathbf{R}_{0}$ :

$$
\mathbf{R}_{0}=\left[\mathbf{K}(\mathbf{0})+E_{0} \mathbf{K}+\Omega_{0} \mathbf{M}\right] \mathbf{U}_{0}
$$

- Linear operators $\mathbf{L}_{\mathbf{t}}$ and $\mathbf{L}$ :

$$
\begin{aligned}
\mathbf{L}_{\mathbf{t}}\left(\mathbf{X}_{0}\right) \boldsymbol{\Delta} \mathbf{X} & =\Delta \Omega \mathbf{M U}_{0}+\left[\mathbf{K}(\mathbf{0})+E_{0} \mathbf{K}+\Omega_{0} \mathbf{M}\right] \Delta \mathbf{U} \\
\mathbf{L}\left(\mathbf{X}_{0}\right) \boldsymbol{\Delta} \mathbf{X} & =\Delta E \mathbf{K U}_{0}
\end{aligned}
$$

- Quadratic operator Q:

$$
\mathbf{Q}(\Delta \mathbf{X}, \Delta \mathbf{X})=\Delta E \mathbf{K} \Delta \mathbf{U}+\Delta \Omega \mathbf{M} \Delta \mathbf{U}
$$

Next, a homotopy parameter $\epsilon$, varying between " 0 " and " 1 ", is judiciously introduced into the non-linear problem (6)[27]. When $\epsilon$ is equal to " 0 ", the problem is a simple one. When $\epsilon$ is equal to " 1 ", the problem is the quadratic problem (6). This parameter is introduced as proposed in [15]:

$$
\mathbf{L}_{\mathbf{t}}\left(\mathbf{X}_{0}\right) \boldsymbol{\Delta} \mathbf{X}+\epsilon \mathbf{L}\left(\mathbf{X}_{0}\right) \boldsymbol{\Delta} \mathbf{X}+\mathbf{Q}(\boldsymbol{\Delta} \mathbf{X}, \boldsymbol{\Delta} \mathbf{X})=-\epsilon \mathbf{R}_{0}
$$

Unknowns are searched as truncated integro-power series. The perturbation parameter is the homotopy parameter $\epsilon$ :

$$
\Delta \mathbf{X}=\sum_{p=1}^{N} \epsilon^{p} \mathbf{X}_{p}
$$

where $N$ is the order of truncation of the polynomial expansion. Moreover, in order to keep a problem properly posed, the following orthogonality condition is added:

$$
\mathbf{U}^{t} \cdot \mathbf{M} \cdot \mathbf{U}_{\text {und }}=0
$$

This choice is the same as in [17], and has already led to accurate results in studies about vibrations of viscoelastic shells.

Inserting the expansions (14) in (13) and adding the orthogonality condition (15), a set of linear problems coming from the identification of the identical powers in $\epsilon$ is obtained:

$$
\left\{\begin{aligned}
\mathbf{L}_{\mathbf{t}}\left(\mathbf{X}_{0}\right) \cdot \mathbf{X}_{p} & =\mathbf{F}_{p} \\
\mathbf{U}_{p}^{t} \cdot \mathbf{M} \cdot \mathbf{U}_{\mathrm{und}} & =0
\end{aligned}\right.
$$

where the right-hand side $\mathbf{F}_{p}$ is defined by:

$$
\mathbf{F}_{p}= \begin{cases}-\mathbf{R}_{0} & p=1 \\ \mathbf{L}\left(\mathbf{X}_{0}\right) \mathbf{X}_{p-1}-\sum_{r=1}^{p-1} \mathbf{Q}\left(\mathbf{X}_{r}, \mathbf{X}_{p-r}\right) & p \geq 2\end{cases}
$$


Initial point $\left(\mathbf{U}_{0}, \Omega_{0}\right)$ at the first iteration is chosen as the solution of the undamped problem $\left(\mathbf{U}_{\text {und }}, \Omega_{\text {und }}\right)$ as proposed in [17]. The right-hand side at the order $p$ depends on the solution of the undamped problem and on the solutions of the $p-1$ previous order exclusively.

All the linear problems have the same tangent operator $\mathbf{L}_{\mathbf{t}}\left(\mathbf{X}_{0}\right)$. This operator is computed only once per iteration and kept for all the orders, reducing the computational time. Thanks to a judicious introduction of the homotopy parameter, Young's modulus becomes a secondary unknown and can be computed with the results obtained at the previous orders.

Unknowns $\mathbf{X}$ are complex values so the system (16) is rewritten separating real $\left({ }^{R}\right)$ parts from imaginary $\left({ }^{I}\right)$ parts:

$$
\left[\begin{array}{cccc}
\mathbf{L}_{\mathbf{t}}^{R} & -\mathbf{L}_{\mathbf{t} \mathbf{U}}^{I} & \mathbf{L}_{\mathbf{t} \boldsymbol{\Omega}}^{R} & -\mathbf{L}_{\mathbf{t} \mathbf{\Omega}}^{I} \\
\mathbf{\mathbf { L } _ { \mathbf { t } } ^ { I } \mathbf { U }} & \mathbf{L}_{\mathbf{t} \mathbf{U}}^{R} & \mathbf{L}_{\mathbf{t} \mathbf{\Omega}}^{I} & \mathbf{L}_{\mathbf{t} \mathbf{\Omega}}^{R} \\
\mathbf{U}_{\text {und }}^{t} \cdot \mathbf{M} & 0 & 0 & 0 \\
0 & \mathbf{U}_{\text {und }}^{t} \cdot \mathbf{M} & 0 & 0
\end{array}\right]\left\{\begin{array}{c}
\mathbf{U}_{p}^{R} \\
\mathbf{U}_{p}^{I} \\
\Omega_{p}^{R} \\
\Omega_{p}^{I}
\end{array}\right\}=\left\{\begin{array}{c}
\mathbf{F}_{p}^{R} \\
\mathbf{F}_{p}^{I} \\
0 \\
0
\end{array}\right\}
$$

where the tangent operator $L_{t} \bullet$ is defined by:

$$
\begin{aligned}
& \mathbf{L}_{\mathbf{t} \mathbf{U}}^{R}=\mathbf{K}(\mathbf{0})+E_{0}^{R} \mathbf{K}+\Omega_{0}^{R} \mathbf{M} \\
& \mathbf{L}_{\mathbf{t} \boldsymbol{\Omega}}^{R}=\mathbf{M} \mathbf{U}_{0}^{R} \\
& \mathbf{L}_{\mathbf{t} \mathbf{U}}^{I}=E_{0}^{I} \mathbf{K}+\Omega_{0}^{I} \mathbf{M} \\
& \mathbf{L}_{\mathbf{t} \boldsymbol{\Omega}}^{I}=\mathbf{M} \mathbf{U}_{0}^{I}
\end{aligned}
$$

and the right-hand side $\mathbf{F}_{p}$ is:

$$
\begin{gathered}
\mathbf{F}_{p}^{R}= \begin{cases}-\left[\mathbf{K}(\mathbf{0})+E_{0}^{R} \mathbf{K}+\Omega_{0}^{R} \mathbf{M}\right] \mathbf{U}_{0}^{R}+\left[E_{0}^{I} \mathbf{K}+\Omega_{0}^{I} \mathbf{M}\right] \mathbf{U}_{0}^{I} & p=1 \\
-E_{p-1}^{R} \mathbf{K} \mathbf{U}_{0}^{R}+E_{p-1}^{I} \mathbf{K} \mathbf{U}_{0}^{I}-\sum_{r=1}^{p-r}\left\{\left[E_{r}^{R} \mathbf{K}+\Omega_{r}^{R} \mathbf{M}\right] \mathbf{U}_{p-r}^{R}-\left[E_{r}^{I} \mathbf{K}+\Omega_{r}^{I} \mathbf{M}\right] \mathbf{U}_{p-r}^{I}\right\} & p \geq 2\end{cases} \\
\mathbf{F}_{p}^{I}= \begin{cases}-\left[\mathbf{K}(\mathbf{0})+E_{0}^{R} \mathbf{K}+\Omega_{0}^{R} \mathbf{M}\right] \mathbf{U}_{0}^{I}-\left[E_{0}^{I} \mathbf{K}+\Omega_{0}^{I} \mathbf{M}\right] \mathbf{U}_{0}^{R} & p=1 \\
-E_{p-1}^{R} \mathbf{K} \mathbf{U}_{0}^{I}-E_{p-1}^{I} \mathbf{K} \mathbf{U}_{0}^{R}-\sum_{r=1}^{p-r}\left\{\left[E_{r}^{R} \mathbf{K}+\Omega_{r}^{R} \mathbf{M}\right] \mathbf{U}_{p-r}^{I}+\left[E_{r}^{I} \mathbf{K}+\Omega_{r}^{I} \mathbf{M}\right] \mathbf{U}_{p-r}^{R}\right\} & p \geq 2\end{cases}
\end{gathered}
$$

Resolution of the system (18) gives unknowns $\mathbf{U}_{p}^{R}, \mathbf{U}_{p}^{I}, \Omega_{p}^{R}, \Omega_{p}^{I}$ for each order $p$. Young's modulus $E_{p}^{R}$ and $E_{p}^{I}$ are computed with the equations established in the next section (cf. 3.2). The final solution of the non-linear problem is obtained by setting $\epsilon=1$ in (13):

$$
\mathbf{X}_{\text {poly }}(\epsilon=1)=\mathbf{X}_{0}+\sum_{p=1}^{N} \mathbf{X}_{p}
$$

In order to accelerate convergence, polynomial representation can be replaced by Padé representation:

$$
\mathbf{X}_{\text {Padé }}(\epsilon=1)=\mathbf{X}_{0}+\sum_{p=1}^{M} \frac{\Delta_{M-p}(\epsilon)}{\Delta_{M}(\epsilon)} \mathbf{X}_{p} \quad \text { with } M=N-1
$$

Polynomials $\Delta_{k}$ are defined as in [28].

In order to check convergence of the iterative process, the following criterion is defined using the residual vector $\mathbf{R}^{(k)}$ :

$$
\sqrt{\mathbf{R}^{(k)} \cdot \mathbf{R}^{(k)}} \leq \text { tolerance }
$$


where the complex residual vector $\mathbf{R}^{(k)}$ is estimated at the end of each iteration:

$$
\mathbf{R}^{(k)}=\left[\mathbf{K}(\mathbf{0})+E(\omega)^{(k)} \mathbf{K}+\Omega^{(k)} \mathbf{M}\right] \mathbf{U}^{(k)}
$$

For each iteration, a choice is made between polynomial (26) or Padé (27) representation. The representation with the lowest residual vector norm (29) is selected, considering the "best order" among the $N$ orders calculated [15].

When this criterion is satisfied, the iterative process is stopped. Then, damped pulsations $\Omega_{a}$ and structural damping $\eta_{m}$ are estimated from the real and imaginary parts of the angular frequency:

$$
\begin{gathered}
\Omega_{a}=\sqrt{\omega^{R 2}-\omega^{I 2}} \\
\eta_{m}=\frac{2 \omega^{R} \omega^{I}}{\omega^{R 2}-\omega^{I 2}}
\end{gathered}
$$

If criterion (28) is not validated, a new iteration is computed with an initial point corresponding to the solution of the previous iteration:

$$
\mathbf{X}_{0}^{(k+1)}=\mathbf{X}^{(k)}
$$

FIGURE 1 describes the procedure of the High Order Newton solver proposed in this subsection.

\subsection{Viscoelastic constitutive laws}

In this paper, two viscoelastic constitutive laws are studied: the hysteretic model (33) and the generalized Maxwell model (34). However, the theoretical development presented in the previous section are not limited to these two constitutive laws. Other viscoelastic laws, such as Zener's model, Kelvin-Voigt generalized model or Golla-Hugues-Mac Tavish model could have been considered [29] [30].

The expressions of the two considered constitutive laws are as follows:

- Hysteretic model:

$$
E(\omega)=E_{0}\left(1+i \eta_{c}\right)
$$

where $E_{0}$ is the real elastic modulus and $\eta_{c}$ is the loss factor.

- Generalized Maxwell model:

$$
E(\omega)=k_{0}+\eta_{0} i \omega+\sum_{j=1}^{N_{\max }} \frac{i \omega}{\left(\frac{i \omega}{k_{j}}+\frac{1}{\eta_{j}}\right)}
$$

where $N_{\max }$ is the number of Maxwell elements. Parameters $k_{j}, \eta_{j}$ depend on the material properties.

Resolution of the matrix system (18) requires expressions of real parts $E_{p}^{R}$ and imaginary parts $E_{p}^{I}$ of the Young's modulus for every order $p(0 \leq p \leq N)$. For the hysteretic model, Young's modulus is constant, so only the order $p=0$ is not null:

$$
\begin{gathered}
E_{p}^{R}=\left\{\begin{array}{l}
E_{0} \text { if } p=0 \\
0 \text { if } p \geq 1
\end{array}\right. \\
E_{p}^{I}=\left\{\begin{array}{l}
E_{0} \eta_{c} \text { if } p=0 \\
0 \text { if } p \geq 1
\end{array}\right.
\end{gathered}
$$

For the generalized Maxwell's model, Young's modulus depends on the frequency and the temperature. The expressions of real and imaginary parts are those proposed by [31] and [32]. 
Calculation of the Young's modulus requires the real and imaginary parts of the angular frequency $\omega^{R}$ and $\omega^{I}$, defined by:

$$
\begin{aligned}
\omega_{p}^{R}=-\frac{\left[\Omega_{p}^{R}+\sum_{r=1}^{p-1}\left(\omega_{r}^{R} \omega_{p-r}^{R}-\omega_{r}^{I} \omega_{p-r}^{I}\right)\right] \omega_{0}^{R}+\left[\Omega_{p}^{I}+\sum_{r=1}^{p-1}\left(\omega_{r}^{R} \omega_{p-r}^{I}+\omega_{r}^{I} \omega_{p-r}^{R}\right)\right] \omega_{0}^{I}}{2\left(\omega_{0}^{R 2}+\omega_{0}^{I 2}\right)} \\
\omega_{p}^{I}=-\frac{\left[\Omega_{p}^{R}+\sum_{r=1}^{p-1}\left(\omega_{r}^{R} \omega_{p-r}^{R}-\omega_{r}^{I} \omega_{p-r}^{I}\right)\right] \omega_{0}^{I}-\left[\Omega_{p}^{I}+\sum_{r=1}^{p-1}\left(\omega_{r}^{R} \omega_{p-r}^{I}+\omega_{r}^{I} \omega_{p-r}^{R}\right)\right] \omega_{0}^{R}}{2\left(\omega_{0}^{R 2}+\omega_{0}^{I 2}\right)}
\end{aligned}
$$




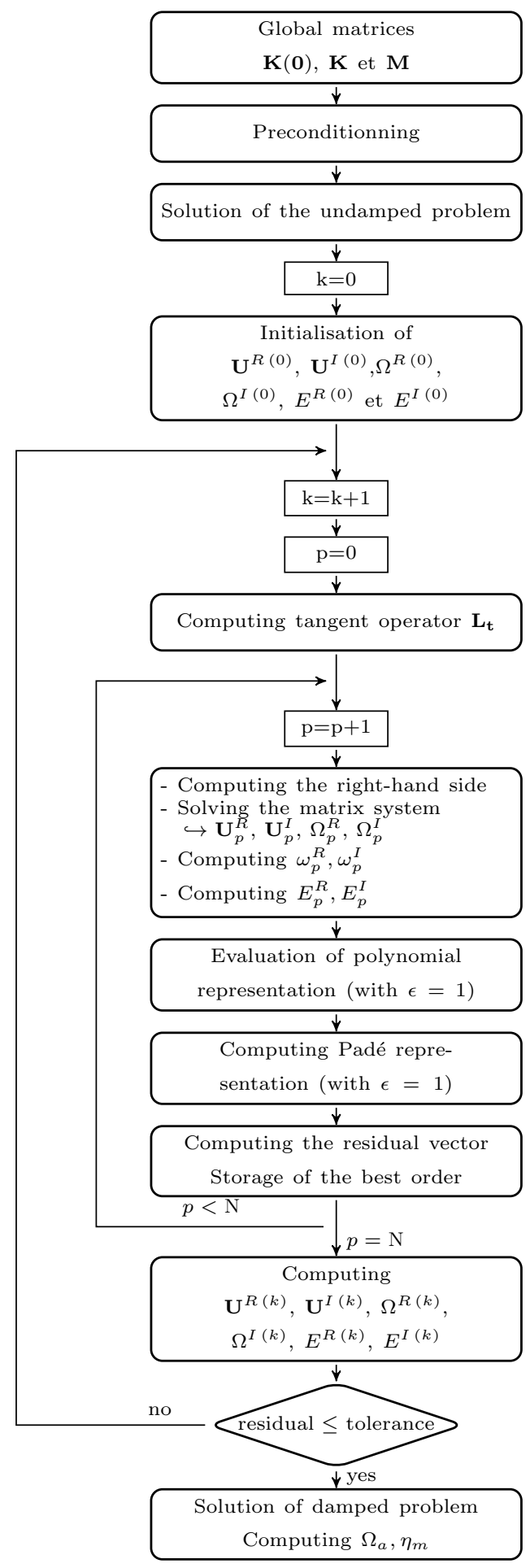

Figure 1: Algorithm of the High Order Newton solver. 


\title{
4. Numerical results
}

In order to validate and illustrate efficiency of the proposed method, results obtained with the High Order Newton solver are compared with those obtained with the classical Newton's method on several problems based on quite simple geometry (square cavity) with multi-component materials.

\subsection{Test case definition}

A square sandwich cavity filled with an inviscid compressible fluid is considered. Geometry and material characteristics are presented on FiguRE 2. It is a test case inspired by [33], already used in previous studies about undamped vibroacoustics interior problems [24] [25].

In order to introduce damping, the sandwich structure is made of a viscoelastic core between two external elastic layers. The viscoelastic behavior is modelled with the hysteretic model (33), a simple model with a constant Young's modulus or the Maxwell generalized model (34), depending on angular frequency and temperature. For the hysteretic model, real elastic modulus is $6.910^{10} \mathrm{~Pa}$ and hysteretic damping $\eta_{c}$ varies between 0.1 and 1.5. Concerning the generalized Maxwell model, in this study, the number of Maxwell elements is very large $\left(N_{\max }=129\right)$, and parameters $\left(k_{j}, \eta_{j}\right)$ are those used in [17]. All these data come from vibration experiments on a polymer carried out by the Arcelor company [31]. Indeed, the objective of the present work is not an experimental study with characterization of a new material. That's why this set of parameters, already available in the research team [31] [17], is used to carry out numerical applications. However, 129 is a very large number of parameters and a set of ten couples $\left(k_{j}, \eta_{j}\right)$ would have been sufficient.

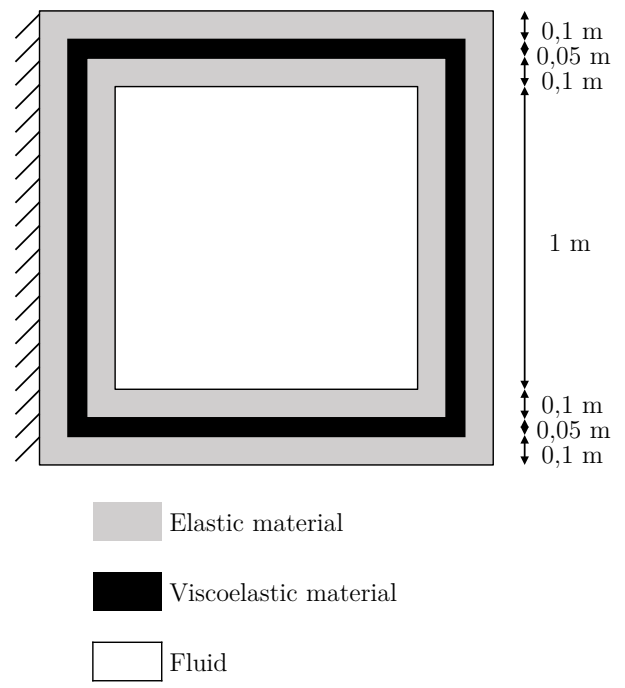

\author{
Elastic material: aluminium \\ $E_{a}=6.9 \times 10^{10} \mathrm{~Pa}$ \\ $\rho_{a}=2766 \mathrm{~kg} . \mathrm{m}^{-3}$ \\ $\nu_{a}=0.3$ \\ thickness: $0.1 \mathrm{~m}$
}

Viscoelastic material: polymer

$E_{0}=1.794 \times 10^{6} \mathrm{~Pa}$

$\rho_{v}=968.1 \mathrm{~kg} \cdot \mathrm{m}^{-3}$

$\nu_{v}=0.3$

thickness: $0.05 \mathrm{~m}$

Fluid:

air: $c_{F}=340 \mathrm{~m} \cdot \mathrm{s}^{-1}, \rho_{F}=1 \mathrm{~kg} \cdot \mathrm{m}^{-3}$

water: $c_{F}=1430 \mathrm{~m} \cdot \mathrm{s}^{-1}, \rho_{F}=1000 \mathrm{~kg} \cdot \mathrm{m}^{-3}$

Figure 2: Geometry and material characteristics of the studied sandwich cavity.

Finite element discretization uses linear quadrilateral elements for the whole system (fluid and structure domains). Fluid elements have four degrees of freedom (pressure at each node) and solid elements have eight degrees of freedom (two displacements at each node). At the fluid-structure interface, nodes are described by three degrees of freedom (one pressure and two displacements). In order to take the "zig-zag" behavior of the sandwich structure into account, it is necessary to discretize the viscoelastic layer with a sufficient number of elements. Convergence tests show that a mesh with 10 elements in the viscoelastic layer, 5 elements in elastic layers and $50 \times 50$ elements in the fluid domain is a reasonable choice. The final mesh has 8100 elements, leading to 8281 nodes. This represents 14361 degrees of freedom for the undamped problem and 28722 degrees of freedom for the damped problem (the number of d.o.f. is doubled due to the complex values). 
For both Newton and HON solvers, the tolerance in the stopping criterion of convergence (28) is chosen equal to $10^{-3}$. The maximal truncation order in the HON solver is $N=20$. All orders are estimated but only the one with the lowest norm of the residual vector (29) is considered, as mentioned in section 3.1.

160

\subsection{Initial point}

An initial point is needed to solve the damped problem. It is chosen as the solution of the associated undamped problem [17], in other words the solution of the problem described FIGURE 2, but with an elastic law instead of a viscoelastic one for the intermediate layer of the sandwich structure. This solution is obtained with the ANM indicator solver proposed in [24] and [25]. Undamped angular frequencies obtained are those presented in TABLE 1. Modes 2 and 7 are presented on Figure 3.

In comparison with the modes obtained for an empty sandwich cavity and for a fluid domain in a rigid cavity, one can identify three different "types" of modes: solid, fluid or coupled. For the cavity filled with air, which is a light fluid, coupling is weak so the modes are nearly the same as for an empty sandwich cavity (solid mode) or for the fluid in rigid cavity (fluid mode). On the contrary, with the cavity filled with water (heavy fluid), the added-mass effect is more significant. FSI coupling is stronger and all the modes are coupled.

\begin{tabular}{ccc}
\hline Mode & Air & Water \\
\hline 1 & $200.70(\mathrm{~S})$ & $170.89(\mathrm{C})$ \\
2 & $323.26(\mathrm{~S})$ & $252.32(\mathrm{C})$ \\
3 & $336.53(\mathrm{~S})$ & $300.70(\mathrm{C})$ \\
4 & $460.48(\mathrm{~S})$ & $424.04(\mathrm{C})$ \\
5 & $793.13(\mathrm{~S})$ & $653.51(\mathrm{C})$ \\
6 & $1014.12(\mathrm{~S})$ & $933.81(\mathrm{C})$ \\
7 & $1068.65(\mathrm{~F})$ & $1061.65(\mathrm{C})$ \\
8 & $1068.66(\mathrm{~F})$ & $1590.93(\mathrm{C})$ \\
9 & $1278.05(\mathrm{~S})$ & $1740.82(\mathrm{C})$ \\
10 & $1511.20(\mathrm{~F})$ & $1894.09(\mathrm{C})$ \\
\hline
\end{tabular}

Table 1: Undamped pulsations $\omega_{\text {und }}$ of the fluid filled sandwich cavity $\left(\operatorname{rad} . \mathrm{s}^{-1}\right)$. Indication (F), (S) or (C) mark fluid modes, solid modes and coupled modes respectively. 

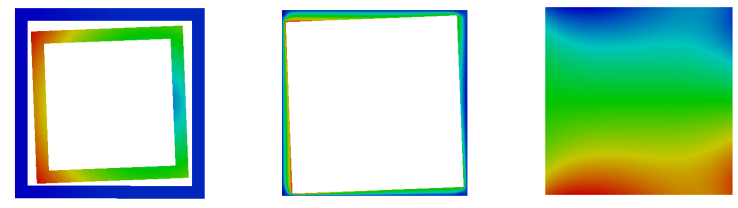

(a) Cavity filled with air - Mode 2 (solid) $\Omega_{\mathrm{und}}=323.26 \mathrm{rad} . \mathrm{s}^{-1}$.
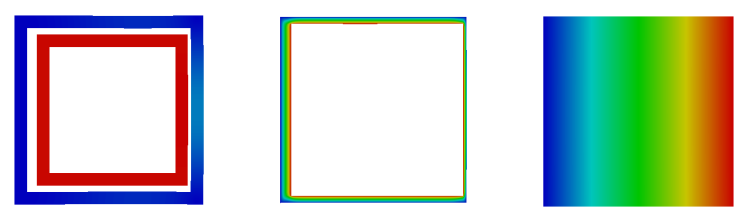

(c) Cavity filled with water - Mode 2 (coupled) $\Omega_{\mathrm{und}}=252.32 \mathrm{rad} . \mathrm{s}^{-1}$.
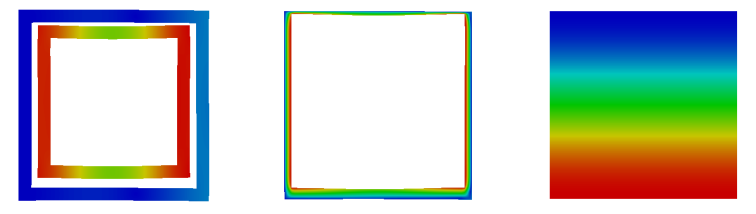

(b) Cavity filled with air - Mode 7 (fluid) $\Omega_{\mathrm{und}}=1068.65{\mathrm{rad} . \mathrm{s}^{-1}}^{-}$.
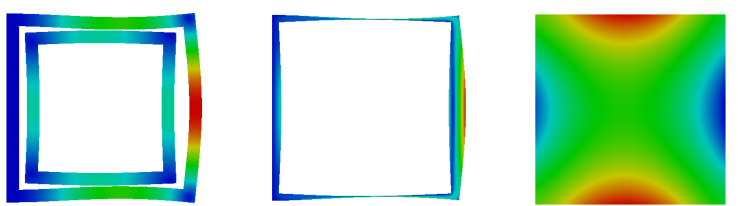

(d) Cavity filled with water - Mode 7 (coupled) $\Omega_{\text {und }}=1061.65$ rad.s ${ }^{-1}$.

Figure 3: Initial undamped modes 2 and 7 of the cavity filled with air or water.

\subsection{Validation of the proposed method}

The first ten damped pulsations, damped modes and structural dampings are computed with Newton's method and HON solver. TABLE 2 and TABLE 3 present results obtained for a cavity filled with air or water, with hysteretic or generalized Maxwell models. Results given in these tables show a good agreement between the proposed method (HON, HON + Padé) and the reference one (Newton), even in the case of a strongly non-linear problem (cf. TABLE 3 ). The small differences (less than $1 \%$ relative difference) are due to the convergence criterion used. Indeed, a "global" convergence criterion, based on the residual vector, is used (28). The use of a "local" criterion, based on the damped pulsation $\Omega_{a}$ and the structural damping $\eta_{m}$ would have reduced the differences between the results obtained by the different solvers:

$$
\left\{\begin{array}{l}
\frac{\eta_{m}^{(k)}-\eta_{m}^{(k-1)}}{\eta_{m}^{(k-1)}} \leq \text { tolerance } \\
\frac{\Omega_{a}^{(k)}-\Omega_{a}^{(k-1)}}{\Omega_{a}^{(k-1)}} \leq \text { tolerance }
\end{array}\right.
$$


(a) Cavity filled with air

\begin{tabular}{ccccccc}
\hline & \multicolumn{2}{c}{ Newton (reference) } & \multicolumn{2}{c}{ HON } & \multicolumn{2}{c}{ HON + Padé } \\
Mode & $\Omega_{a}\left(\right.$ rad.s $\left.^{-1}\right)$ & $\eta_{m}$ & $\Omega_{a}\left(\right.$ rad.s $\left.^{-1}\right)$ & $\eta_{m}$ & $\Omega_{a}\left(\right.$ rad.s $\left.^{-1}\right)$ & $\eta_{m}$ \\
\hline $1(\mathrm{~S})$ & 204.15 & $1.4610^{-1}$ & 204.15 & $1.4610^{-1}$ & 204.15 & $1.4610^{-1}$ \\
$2(\mathrm{~S})$ & 323.61 & $4.9310^{-1}$ & 323.61 & $4.9310^{-1}$ & 323.61 & $4.9310^{-1}$ \\
$3(\mathrm{~S})$ & 338.47 & $4.7010^{-1}$ & 338.47 & $4.7010^{-1}$ & 338.47 & $4.7010^{-1}$ \\
$4(\mathrm{~S})$ & 461.06 & $4.0510^{-1}$ & 461.06 & $4.0510^{-1}$ & 461.06 & $4.0510^{-1}$ \\
$5(\mathrm{~S})$ & 792.79 & $8.4610^{-2}$ & 792.79 & $8.4610^{-2}$ & 792.79 & $8.4610^{-2}$ \\
$6(\mathrm{~S})$ & 1016.69 & $5.3910^{-2}$ & 1016.69 & $5.3910^{-2}$ & 1016.69 & $5.3910^{-2}$ \\
$7(\mathrm{~F})$ & 1068.65 & $4.3210^{-5}$ & 1068.65 & $4.3210^{-5}$ & 1068.65 & $4.3210^{-5}$ \\
$8(\mathrm{~F})$ & 1068.65 & $6.5110^{-5}$ & 1068.65 & $6.5110^{-5}$ & 1068.65 & $6.5110^{-5}$ \\
$9(\mathrm{~S})$ & 1276.60 & $7.1810^{-2}$ & 1276.60 & $7.1810^{-2}$ & 1276.60 & $7.1810^{-2}$ \\
$10(\mathrm{~F})$ & 1511.21 & $2.2810^{-5}$ & 1511.21 & $2.2810^{-5}$ & 1511.21 & $2.2810^{-5}$ \\
\hline
\end{tabular}

(b) Cavity filled with water

\begin{tabular}{ccccccc}
\hline & \multicolumn{2}{c}{ Newton (reference) } & \multicolumn{2}{c}{ HON } & \multicolumn{2}{c}{ HON + Padé } \\
Mode & $\Omega_{a}\left(\right.$ rad.s $\left.^{-1}\right)$ & $\eta_{m}$ & $\Omega_{a}\left({\left.\mathrm{rad} . \mathrm{s}^{-1}\right)}^{-1}\right.$ & $\eta_{m}$ & $\Omega_{a}\left(\mathrm{rad.s}^{-1}\right)$ & $\eta_{m}$ \\
\hline $1(\mathrm{C})$ & 174.70 & $1.8710^{-1}$ & 174.70 & $1.8710^{-1}$ & 174.70 & $1.8710^{-1}$ \\
$2(\mathrm{C})$ & 253.61 & $4.7310^{-1}$ & 253.61 & $4.7310^{-1}$ & 253.61 & $4.7310^{-1}$ \\
$3(\mathrm{C})$ & 301.40 & $4.8110^{-1}$ & 301.40 & $4.8110^{-1}$ & 301.40 & $4.8110^{-1}$ \\
$4(\mathrm{C})$ & 424.55 & $3.6610^{-1}$ & 424.55 & $3.6610^{-1}$ & 424.55 & $3.6610^{-1}$ \\
$5(\mathrm{C})$ & 652.57 & $8.8310^{-2}$ & 652.57 & $8.8310^{-2}$ & 652.57 & $8.8310^{-2}$ \\
$6(\mathrm{C})$ & 934.13 & $1.7510^{-2}$ & 934.13 & $1.7510^{-2}$ & 934.13 & $1.7510^{-2}$ \\
$7(\mathrm{C})$ & 1061.59 & $1.0610^{-1}$ & 1061.59 & $1.0610^{-1}$ & 1061.59 & $1.0610^{-1}$ \\
$8(\mathrm{C})$ & 1595.88 & $3.3310^{-2}$ & 1595.88 & $3.3310^{-2}$ & 1595.88 & $3.3310^{-2}$ \\
$9(\mathrm{C})$ & 1743.68 & $3.0810^{-2}$ & 1743.68 & $3.0810^{-2}$ & 1743.68 & $3.0810^{-2}$ \\
$10(\mathrm{C})$ & 1990.79 & $3.8510^{-2}$ & 1990.79 & $3.8510^{-2}$ & 1990.79 & $3.8510^{-2}$ \\
\hline
\end{tabular}

Table 2: Hysteretic model, $\eta_{c}=0,5$ - Damped pulsations $\Omega_{a}$ and structural damping $\eta_{m}$ obtained by Newton's method and HON solver (with or without Padé representation). Results obtained with Newton's method are considered as the reference. Indication $(\mathrm{F}),(\mathrm{S})$ or $(\mathrm{C})$ mark fluid modes, solid modes and coupled modes respectively. 
(a) Cavity filled with air

\begin{tabular}{ccccccc}
\hline & \multicolumn{2}{c}{ Newton (reference) } & \multicolumn{2}{c}{ HON } & \multicolumn{2}{c}{ HON + Padé } \\
Mode & $\Omega_{a}\left(\right.$ rad.s $\left.^{-1}\right)$ & $\eta_{m}$ & $\Omega_{a}\left(\right.$ rad.s $\left.^{-1}\right)$ & $\eta_{m}$ & $\Omega_{a}\left(\right.$ rad.s $\left.^{-1}\right)$ & $\eta_{m}$ \\
\hline $1(\mathrm{~S})$ & 218.54 & $8.2410^{-2}$ & 218.54 & $8.2010^{-2}$ & 218.54 & $8.2010^{-2}$ \\
$2(\mathrm{~S})$ & 425.07 & $4.7410^{-1}$ & 428.87 & $4.4310^{-1}$ & 428.98 & $4.4110^{-1}$ \\
$3(\mathrm{~S})$ & 441.81 & $4.3610^{-1}$ & 444.81 & $4.0810^{-1}$ & 444.81 & $4.0810^{-1}$ \\
$4(\mathrm{~S})$ & 598.06 & $4.7610^{-1}$ & 603.27 & $4.4210^{-1}$ & 603.65 & $4.4210^{-1}$ \\
$5(\mathrm{~S})$ & 859.98 & $1.7810^{-1}$ & 860.49 & $1.7710^{-1}$ & 860.49 & $1.7710^{-1}$ \\
$6(\mathrm{~S})$ & 1074.29 & $8.6410^{-2}$ & 1074.36 & $8.6210^{-2}$ & 1074.36 & $8.6210^{-2}$ \\
$7(\mathrm{~F})$ & 1068.70 & $1.4910^{-4}$ & 1068.70 & $1.4910^{-4}$ & 1068.70 & $1.4910^{-4}$ \\
$8(\mathrm{~F})$ & 1068.61 & $1.6910^{-4}$ & 1068.61 & $1.6910^{-4}$ & 1068.61 & $1.6910^{-4}$ \\
$9(\mathrm{~S})$ & 1394.33 & $2.1610^{-1}$ & 1394.33 & $2.1610^{-1}$ & 1395.92 & $2.1510^{-1}$ \\
$10(\mathrm{~F})$ & 1511.25 & $5.9210^{-5}$ & 1511.25 & $5.9210^{-5}$ & 1511.25 & $5.9210^{-5}$ \\
\hline
\end{tabular}

(b) Cavity filled with water

\begin{tabular}{ccccccc}
\hline & \multicolumn{2}{c}{ Newton (reference) } & \multicolumn{2}{c}{ HON } & \multicolumn{2}{c}{ HON + Padé } \\
Mode & $\Omega_{a}\left({\left.\mathrm{rad} . \mathrm{s}^{-1}\right)}^{-1}\right.$ & $\eta_{m}$ & $\Omega_{a}\left({\left.\mathrm{rad} . \mathrm{s}^{-1}\right)}^{-1}\right.$ & $\eta_{m}$ & $\Omega_{a}\left({\left.\mathrm{rad} . \mathrm{s}^{-1}\right)}\right.$ & $\eta_{m}$ \\
\hline $1(\mathrm{C})$ & 189.39 & $9.3410^{-2}$ & 189.39 & $9.2910^{-2}$ & 189.39 & $9.2910^{-2}$ \\
$2(\mathrm{C})$ & 326.00 & $3.7110^{-1}$ & 327.43 & $3.5210^{-1}$ & 327.43 & $3.5210^{-1}$ \\
$3(\mathrm{C})$ & 392.45 & $4.3710^{-1}$ & 395.00 & $4.0910^{-1}$ & 395.52 & $4.0810^{-1}$ \\
$4(\mathrm{C})$ & 539.02 & $4.0410^{-1}$ & 544.29 & $3.7710^{-1}$ & 542.64 & $3.8110^{-1}$ \\
$5(\mathrm{C})$ & 705.56 & $1.8010^{-1}$ & 705.98 & $1.7910^{-1}$ & 705.98 & $1.7910^{-1}$ \\
$6(\mathrm{C})$ & 952.00 & $3.8210^{-2}$ & 952.01 & $3.8210^{-2}$ & 952.01 & $3.8210^{-2}$ \\
$7(\mathrm{C})$ & 1188.55 & $2.4310^{-1}$ & 1190.69 & $2.4110^{-1}$ & 1190.41 & $2.4110^{-1}$ \\
$8(\mathrm{C})$ & 1663.80 & $8.2210^{-2}$ & 1663.89 & $8.2110^{-2}$ & 1663.89 & $8.2110^{-2}$ \\
$9(\mathrm{C})$ & 1818.86 & $8.8010^{-2}$ & 1818.99 & $8.7810^{-2}$ & 1818.99 & $8.7810^{-2}$ \\
$10(\mathrm{C})$ & 2028.73 & $4.7410^{-2}$ & 2028.92 & $4.6610^{-2}$ & 2028.81 & $4.7010^{-2}$ \\
\hline
\end{tabular}

Table 3: Generalized Maxwell model, $T=30^{\circ} \mathrm{C}$ - Damped pulsations $\Omega_{a}$ and structural damping $\eta_{m}$ obtained by Newton's method and HON solver (with or without Padé representation). Results obtained with Newton's method are considered as the reference. Indication (F), (S) or (C) mark fluid modes, solid modes and coupled modes respectively.

\subsection{Comparison of the numerical methods performances}

In this section, performances of the HON solver are compared with those of the classical Newton's method. Efficiency of the HON solver is highlighted considering the number of iterations required to compute the damped pulsations and damped modes. Next, the truncation order at convergence is studied to show interest of Padé representation.

TABLE 4 and TABLE 5 present the number of iterations required to compute the damped modes 2 and 7 . Whatever the viscoelastic model and the type of mode studied, the HON solver is more efficient than the Newton's method. Complexity of the generalized Maxwell model makes the convergence difficult with the classical Newton's method. On the contrary, convergence is obtained with only a few iterations with the HON solver (3 iterations in our tests). Using Padé representation, convergence is obtained with only one or two iterations. This shows the interest of the HON solver and Padé representation to compute damped pulsations in the case of non-linear fluid-structure interaction problems. Indeed, in contrast to the Newton solver where non-linear terms are neglected, the HON solver takes into account these non-linear terms, which reduces the number of iterations. Moreover, the Padé representation is used as a convergence accelerator, which increases the solver efficiency.

Convergence speed depends on the type of mode (fluid/solid/coupled) and on the level of damping introduced. It appears that the convergence of fluid modes is faster than the convergence of solid and coupled modes. Indeed, fluid modes are weakly damped so the damped mode is very close to the initial 
point.

For solid and coupled modes, convergence is slower when damping increases. Indeed, when there is strong damping, the problem is highly non-linear and more complicated to solve. For the generalized Maxwell model, the studied viscoelastic material is very viscous when temperature is low and slightly viscous when temperature is high. So damping is more important at low temperatures than at high temperatures. This is why convergence is faster when temperature increases.

TABLE 6 and TABLE 7 present CPU time required to compute the damped modes 2 and 7 . It appears that HON solvers often require less CPU time than Newton solver, especially in the case of Maxwell's model. Differences between HON and HON+Padé solvers can be explained by the computation time of the Padé representation, which requires about $5 \mathrm{~s}$ per iteration. Finally, in some cases, the Newton solver requires less CPU time than the HON solver. This is probably due to the low number of degrees of freedom of the studied problem (less than 30000 dof). In this case, treatment of the right-hand side at high orders can lead to significant computation times compared to a matrix factorization.

(a) Mode 2 (solid) - Cavity filled with air

\begin{tabular}{lccccc}
\hline$\eta_{c}$ & 0.1 & 0.2 & 0.5 & 1.0 & 1.5 \\
\hline Newton & 2 & 3 & 3 & 3 & 3 \\
HON & 1 & 2 & 2 & 2 & 2 \\
HON + Padé & 1 & 1 & 1 & 2 & 2 \\
\hline
\end{tabular}

(c) Mode 2 (coupled) - Cavity filled with water

\begin{tabular}{lccccc}
\hline$\eta_{c}$ & 0.1 & 0.2 & 0.5 & 1.0 & 1.5 \\
\hline Newton & 2 & 2 & 3 & 3 & 3 \\
HON & 1 & 1 & 1 & 2 & 2 \\
HON + Padé & 1 & 1 & 1 & 1 & 1 \\
\hline
\end{tabular}

(b) Mode 7 (fluid) - Cavity filled with air

\begin{tabular}{lccccc}
\hline$\eta_{c}$ & 0.1 & 0.2 & 0.5 & 1.0 & 1.5 \\
\hline Newton & 2 & 2 & 2 & 2 & 2 \\
HON & 1 & 1 & 1 & 1 & 1 \\
HON + Padé & 1 & 1 & 1 & 1 & 1 \\
\hline
\end{tabular}

(d) Mode 7 (coupled) - Cavity filled with water

\begin{tabular}{lccccc}
\hline$\eta_{c}$ & 0.1 & 0.2 & 0.5 & 1.0 & 1.5 \\
\hline Newton & 3 & 3 & 3 & 4 & 4 \\
HON & 1 & 1 & 2 & 2 & 2 \\
HON + Padé & 1 & 1 & 1 & 2 & 2 \\
\hline
\end{tabular}

Table 4: Hysteretic model - Comparison of the number of iterations required to compute the damped modes 2 and 7 . Performances of the HON solver (with or without Padé representation) are compared with those of the classical Newton algorithm.

(a) Mode 2 (solid) - Cavity filled with air

\begin{tabular}{lcccccc}
\hline Temperature $\left({ }^{\circ} \mathrm{C}\right)$ & 20 & 30 & 40 & 50 & 60 & 70 \\
\hline Newton & 16 & 11 & 7 & 7 & 7 & 7 \\
HON & 3 & 2 & 2 & 2 & 2 & 1 \\
HON + Padé & 2 & 2 & 2 & 1 & 1 & 1 \\
\hline
\end{tabular}

(c) Mode 2 (coupled) - Cavity filled with water

\begin{tabular}{lcccccc}
\hline Temperature $\left({ }^{\circ} \mathrm{C}\right)$ & 20 & 30 & 40 & 50 & 60 & 70 \\
\hline Newton & 15 & 10 & 6 & 7 & 7 & 6 \\
HON & 2 & 2 & 2 & 1 & 1 & 1 \\
HON + Padé & 2 & 2 & 1 & 1 & 1 & 1 \\
\hline
\end{tabular}

(b) Mode 7 (fluid) - Cavity filled with air

\begin{tabular}{lcccccc}
\hline Temperature $\left({ }^{\circ} \mathrm{C}\right)$ & 20 & 30 & 40 & 50 & 60 & 70 \\
\hline Newton & 4 & 3 & 3 & 3 & 3 & 3 \\
HON & 2 & 1 & 1 & 1 & 1 & 1 \\
HON + Padé & 1 & 1 & 1 & 1 & 1 & 1 \\
\hline
\end{tabular}

(d) Mode 7 (coupled) - Cavity filled with water

\begin{tabular}{lcccccc}
\hline Temperature $\left({ }^{\circ} \mathrm{C}\right)$ & 20 & 30 & 40 & 50 & 60 & 70 \\
\hline Newton & 15 & 9 & 6 & 5 & 5 & 5 \\
HON & 3 & 2 & 2 & 2 & 2 & 1 \\
HON + Padé & 2 & 2 & 2 & 1 & 1 & 1 \\
\hline
\end{tabular}

Table 5: Generalized Maxwell model - Comparison of the number of iterations required to compute the damped modes 2 and 7. Performances of the HON solver (with or without Padé representation) are compared with those of the classical Newton algorithm. 
(a) Mode 2 (solid) - Cavity filled with air

\begin{tabular}{lccccc}
\hline$\eta_{c}$ & 0.1 & 0.2 & 0.5 & 1.0 & 1.5 \\
\hline Newton & 23 & 52 & 34 & 34 & 33 \\
HON & 20 & 40 & 40 & 40 & 40 \\
HON + Padé & 25 & 25 & 25 & 50 & 49 \\
\hline
\end{tabular}

(c) Mode 2 (coupled) - Cavity filled with water

\begin{tabular}{lccccc}
\hline$\eta_{c}$ & 0.1 & 0.2 & 0.5 & 1.0 & 1.5 \\
\hline Newton & 23 & 33 & 34 & 34 & 33 \\
HON & 20 & 20 & 20 & 40 & 40 \\
HON + Padé & 25 & 25 & 25 & 25 & 24 \\
\hline
\end{tabular}

(b) Mode 7 (fluid) - Cavity filled with air

\begin{tabular}{lccccc}
\hline$\eta_{c}$ & 0.1 & 0.2 & 0.5 & 1.0 & 1.5 \\
\hline Newton & 23 & 35 & 23 & 22 & 22 \\
HON & 20 & 20 & 20 & 20 & 20 \\
HON + Padé & 25 & 25 & 25 & 25 & 25 \\
\hline
\end{tabular}

(d) Mode 7 (coupled) - Cavity filled with water

\begin{tabular}{lccccc}
\hline$\eta_{c}$ & 0.1 & 0.2 & 0.5 & 1.0 & 1.5 \\
\hline Newton & 34 & 49 & 34 & 45 & 44 \\
HON & 20 & 20 & 40 & 40 & 40 \\
HON + Padé & 25 & 25 & 25 & 49 & 49 \\
\hline
\end{tabular}

Table 6: Hysteretic model - Comparison of the CPU time (s) required to compute the damped modes 2 and 7 . Performances of the HON solver (with or without Padé representation) are compared with those of the classical Newton algorithm.

(a) Mode 2 (solid) - Cavity filled with air

\begin{tabular}{lcccccc}
\hline Temperature $\left({ }^{\circ} \mathrm{C}\right)$ & 20 & 30 & 40 & 50 & 60 & 70 \\
\hline Newton & 19 & 131 & 83 & 82 & 82 & 82 \\
HON & 70 & 46 & 46 & 46 & 47 & 23 \\
HON + Padé & 56 & 56 & 56 & 28 & 28 & 28 \\
\hline
\end{tabular}

(c) Mode 2 (coupled) - Cavity filled with water

\begin{tabular}{lcccccc}
\hline Temperature $\left({ }^{\circ} \mathrm{C}\right)$ & 20 & 30 & 40 & 50 & 60 & 70 \\
\hline Newton & 177 & 118 & 71 & 82 & 82 & 70 \\
HON & 46 & 46 & 46 & 23 & 23 & 23 \\
HON + Padé & 56 & 56 & 28 & 32 & 28 & 28 \\
\hline
\end{tabular}

(b) Mode 7 (fluid) - Cavity filled with air

\begin{tabular}{lcccccc}
\hline Temperature $\left({ }^{\circ} \mathrm{C}\right)$ & 20 & 30 & 40 & 50 & 60 & 70 \\
\hline Newton & 5 & 36 & 35 & 35 & 35 & 35 \\
HON & 46 & 23 & 23 & 23 & 23 & 23 \\
HON + Padé & 28 & 28 & 28 & 28 & 28 & 28 \\
\hline
\end{tabular}

(d) Mode 7 (coupled) - Cavity filled with water

\begin{tabular}{lrrrrrr}
\hline Temperature $\left({ }^{\circ} \mathrm{C}\right)$ & 20 & 30 & 40 & 50 & 60 & 70 \\
\hline Newton & 177 & 106 & 71 & 59 & 59 & 59 \\
HON & 70 & 46 & 46 & 46 & 47 & 23 \\
HON + Padé & 56 & 56 & 56 & 32 & 28 & 28 \\
\hline
\end{tabular}

Table 7: Generalized Maxwell model - Comparison of the CPU time (s) required to compute the damped modes 2 and 7. Performances of the HON solver (with or without Padé representation) are compared with those of the classical Newton algorithm.

TABLE 8 and TABLE 9 show the truncation order at convergence for a cavity filled with air or water, considering modes 2 and 7 . It must be noted that the truncation order at convergence is the order $p$ from which the convergence criterion (28) is verified. It may be different from the "best order", which is the order leading to the lowest norm of the residual vector (29). Results in TABLE 8 and TABLE 9 show that convergence slows down when damping increases (i.e. when temperature decreases). First, the number of required order increases. Then, when the $N$ orders are not enough to converge, a new iteration is computed. For example, in TABLE 9, for mode 2 of a cavity filled with water, when the temperature is high $\left(70^{\circ} \mathrm{C}\right)$, only one iteration and 7 terms of the polynomial approximation are required to compute the first ten damped angular frequencies. Then, the number of terms increases $\left(15\right.$ terms when $\left.\mathrm{T}=50^{\circ} \mathrm{C}\right)$ as long as one iteration is not sufficient to obtain convergence. So, when the temperature is lower than $40^{\circ} \mathrm{C}$, convergence requires two iterations ( 2 iterations and 1 term for $\mathrm{T}=40^{\circ} \mathrm{C}$, but 2 iterations and 11 terms for $\mathrm{T}=20^{\circ} \mathrm{C}$ ). So, it may be judicious to choose a truncation order $N$ sufficiently high to reduce the number of iterations. However, in some specific cases, it is not possible to have one iteration only because the first tangent operator gives a poor direction and the computation is divergent. Thus, a second iteration is necessary to achieve convergence.

Moreover, results highlight the advantage of Padé representation. Indeed, the latter allows to decrease both the iteration number and the truncation order. The results of HON + Padé solver show that Padé 
representation is more often chosen than polynomial representation. Indeed, this Padé representation is often the one with the lowest norm of the residual vector.

(a) Mode 2 (solid) - Cavity filled with air

\begin{tabular}{rccccc}
\hline$\eta_{c}$ & 0.1 & 0.2 & 0.5 & 1.0 & 1.5 \\
\hline \multicolumn{5}{c}{ HON } \\
Nb. of it. & 1 & 2 & 2 & 2 & 2 \\
Conv. order & 20 & 1 & 1 & 3 & 4 \\
\hline & \multicolumn{5}{c}{ HON + Padé } \\
\cline { 2 - 6 } Nb. of it. & 1 & 1 & 1 & 2 & 2 \\
Conv. order & 9 & 10 & 20 & 3 & 3 \\
Chosen repr. Padé Padé Padé Poly. Padé \\
\hline
\end{tabular}

(c) Mode 2 (coupled) - Cavity filled with water

\begin{tabular}{rccccc}
\hline$\eta_{c}$ & 0.1 & 0.2 & 0.5 & 1.0 & 1.5 \\
\hline & \multicolumn{5}{c}{ HON } \\
Nb. of it. & 1 & 1 & 1 & 2 & 2 \\
Conv. order & 4 & 4 & 20 & 1 & 1 \\
\hline & \multicolumn{5}{c}{ HON + Padé } \\
\cline { 2 - 6 } Nb. of it. & 1 & 1 & 1 & 1 & 1 \\
Conv. order & 4 & 6 & 9 & 13 & 12 \\
Chosen repr. Poly. Poly. Padé Padé Padé \\
\hline
\end{tabular}

(b) Mode 7 (fluid) - Cavity filled with air

\begin{tabular}{|c|c|c|c|c|c|}
\hline$\eta_{c}$ & 0.1 & 0.2 & 0.5 & 1.0 & 1.5 \\
\hline & \multicolumn{5}{|c|}{ HON } \\
\hline Nb. of it. & 1 & 1 & 1 & 1 & 1 \\
\hline \multirow[t]{2}{*}{ Conv. order } & 3 & 3 & 4 & 6 & 7 \\
\hline & \multicolumn{5}{|c|}{ HON + Padé } \\
\hline Nb. of it. & 1 & 1 & 1 & 1 & 1 \\
\hline & 3 & 4 & 5 & 6 & 8 \\
\hline \multicolumn{6}{|l|}{ Chosen repr. } \\
\hline
\end{tabular}

(d) Mode 7 (coupled) - Cavity filled with water

\begin{tabular}{rccccc}
\hline$\eta_{c}$ & 0.1 & 0.2 & 0.5 & 1.0 & 1.5 \\
\hline \multicolumn{5}{c}{ HON } \\
\cline { 2 - 6 } Nb. of it. & 1 & 1 & 2 & 2 & 2 \\
Conv. order & 7 & 10 & 1 & 5 & 7 \\
\hline & \multicolumn{5}{c}{ HON + Padé } \\
\cline { 2 - 6 } Nb. of it. & 1 & 1 & 1 & 2 & 2 \\
Conv. order & 5 & 7 & 3 & 3 & 3 \\
Chosen repr. Padé Padé Padé Poly. Poly.
\end{tabular}

Table 8: Hysteretic model - Comparison of the iteration number and the truncation order at convergence. For the HON + Padé solver, the chosen representation is specified (polynomial or Padé). 
(a) Mode 2 (solid) - Cavity filled with air

\begin{tabular}{rcccccc}
\hline Temp. $\left({ }^{\circ} \mathrm{C}\right)$ & 20 & 30 & 40 & 50 & 60 & 70 \\
\hline Nb. of it. & 3 & 2 & 2 & 2 & 2 & 1 \\
Conv. order & 1 & 8 & 4 & 1 & 1 & 20 \\
\hline \multicolumn{7}{c}{ HON + Padé } \\
\cline { 2 - 7 } Nb. of it. & 2 & 2 & 2 & 1 & 1 & 1 \\
Conv. order & 14 & 4 & 3 & 20 & 14 & 11 \\
Chosen repr. Padé Padé Poly. Padé Padé Padé
\end{tabular}

(c) Mode 2 (coupled) - Cavity filled with water

\begin{tabular}{rccccccc}
\hline Temp. $\left({ }^{\circ} \mathrm{C}\right)$ & 20 & 30 & 40 & 50 & 60 & 70 \\
\hline & \multicolumn{5}{c}{ HON } \\
\cline { 2 - 7 } Nb. of it. & 2 & 2 & 2 & 1 & 1 & 1 \\
Conv. order & 11 & 4 & 1 & 15 & 9 & 7 \\
\hline \multicolumn{6}{c}{ HON + Padé } \\
\cline { 2 - 7 } Nb. of it. & 2 & 2 & 1 & 1 & 1 & 1 \\
Conv. order & 7 & 3 & 14 & 14 & 9 & 8 \\
Chosen repr. Padé Padé Padé Padé Poly. Padé \\
\hline
\end{tabular}

(b) Mode 7 (fluid) - Cavity filled with air

\begin{tabular}{rrrrrrr}
\hline Temp. $\left({ }^{\circ} \mathrm{C}\right)$ & 20 & 30 & 40 & 50 & 60 & 70 \\
\hline Nb. of it. & 2 & 1 & 1 & 1 & 1 & 1 \\
Conv. order & 3 & 9 & 5 & 5 & 4 & 4 \\
\hline & \multicolumn{5}{c}{ HON + Padé } \\
\cline { 2 - 7 } Nb. of it. & 1 & 1 & 1 & 1 & 1 & 1 \\
Conv. order & 8 & 6 & 6 & 5 & 5 & 5 \\
Chosen repr. Padé Padé Poly. Poly. Padé Poly. \\
\hline
\end{tabular}

(d) Mode 7 (coupled) - Cavity filled with water

\begin{tabular}{rcccccc}
\hline Temp. $\left({ }^{\circ} \mathrm{C}\right)$ & 20 & 30 & 40 & 50 & 60 & 70 \\
\hline Nb. of it. & 3 & 2 & 2 & 2 & 2 & 1 \\
Conv. order & 2 & 9 & 5 & 2 & 1 & 15 \\
\hline & \multicolumn{5}{c}{ HON + Padé } \\
\cline { 2 - 7 } Nb. of it. & 2 & 2 & 2 & 1 & 1 & 1 \\
Conv. order & 5 & 4 & 3 & 13 & 12 & 10 \\
Chosen repr. Padé Padé Poly. Padé Padé Padé \\
\hline
\end{tabular}

Table 9: Generalized Maxwell model - Comparison of the iteration number and the truncation order at convergence. For the HON + Padé solver, the chosen representation is specified (polynomial or Padé).

\subsection{Mechanical behavior of the damped fluid-structure system}

Damping effects on the vibrational behavior of the coupled problem are now studied. TABLE 10 and TABLE 11 present damped angular frequency and structural damping obtained with the hysteretic model, when the viscoelastic damping factor $\eta_{c}$ varies between 0.1 and 1.5. TABLE 12 and TABLE 13 present the results obtained with the generalized Maxwell model, for temperatures varying from $20^{\circ} \mathrm{C}$ to $70^{\circ} \mathrm{C}$.

With a light fluid, like air (cf. TABLE 10 and TABLE 12), the weak coupling generates minor damping effect on the fluid modes. With a heavy fluid, like water (cf. TABLE 11 and TABLE 13), coupling is stronger, and the damping affects every mode. Indeed, damping is introduced in the sandwich structure, that is in the solid domain. In this way, structural damping value is in the range of $10^{-5}$ to $10^{-4}$ for fluid modes, but in the range of $10^{-3}$ to $10^{-1}$ for solid and coupled modes.

Evolutions of damped angular frequencies and structural damping are plotted on Figure 8, FigURE 9, Figure 10 and Figure 11, for the two viscoelastic models and for different types of modes (solid/ fluid/ coupled). For solid and coupled modes, damped angular frequency and structural damping increase with increasing damping (i.e. when hysteretic damping increases or when temperature decreases). For the fluid mode (air, mode 2), hysteretic damping and temperature have no incidence on the damped angular frequency and structural damping is nearly null. These trends are the same as in the literature [17] [8].

Damped modes corresponding to modes 2 and 7 are presented in Figure 4, Figure 5, Figure 6 and FiguRE 7. In order to highlight influence of the viscoelastic layer, the damped modes are compared to the undamped modes (cf. FIgURE 3), used as the initial point in the HON solver. Impact of damping is particularly visible with the cavity filled with water, where fluid and solid domains are strongly coupled (for example see Figure 7(b) and Figure 7(d)). Indeed, the viscoelastic layer enables to transmit more or less vibratory energy from one domain to another depending on the hysteretic loss factor $\eta_{c}$ or on the temperature, in other words depending on the viscosity. 

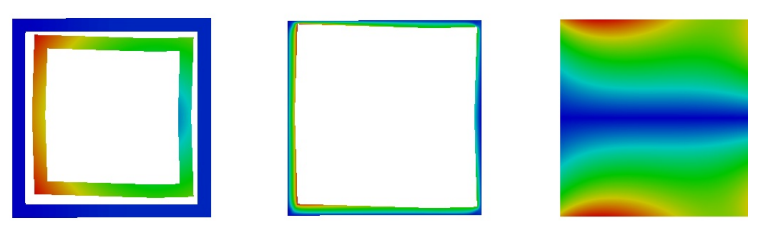

(a) $\eta_{c}=0,1$ - Damped mode 2 (solid) $-\Omega_{a}=323.27$ rad.s ${ }^{-1}$.
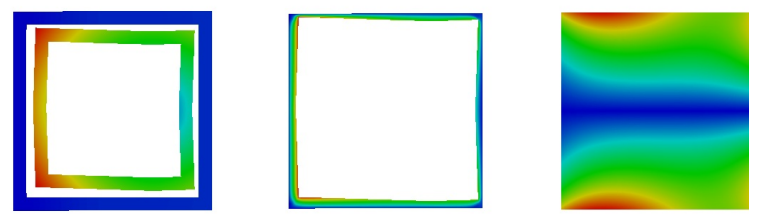

(c) $\eta_{c}=1,5$ - Damped mode 2 (solid) $-\Omega_{a}=353.48$ rad. $\mathrm{s}^{-1}$
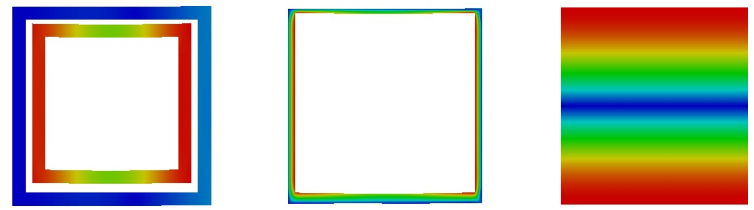

(b) $\eta_{c}=0,1-$ Damped mode 7 (fluid) $-\Omega_{a}=1068.65$ rad.s ${ }^{-1}$
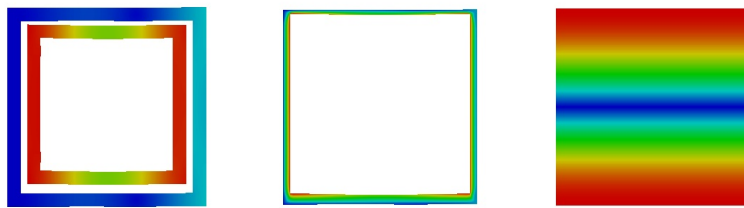

(d) $\eta_{c}=1,5$ - Damped mode 7 (fluid) $-\Omega_{a}=1068.64 \mathrm{rad} . \mathrm{s}^{-1}$.

Figure 4: Hysteretic model - Cavity filled with air - Visualisation of damped modes 2 and 7 with different hysteretic damping factor $\eta_{c}$.
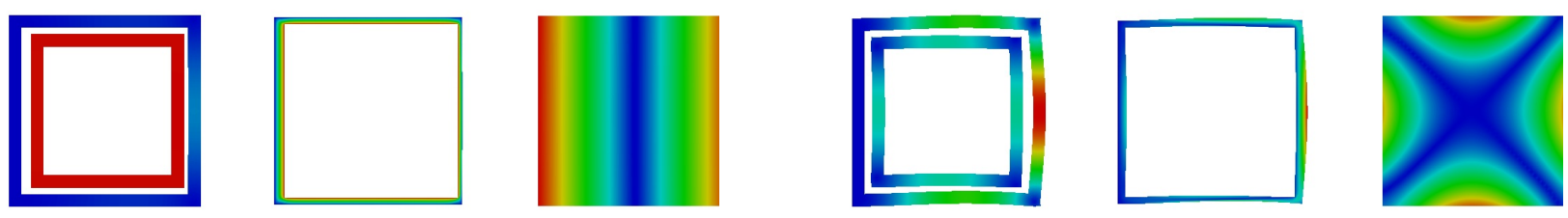

(a) $\eta_{c}=0,1-$ Damped mode 2 (coupled) $-\Omega_{a}=252.37$ rad.s ${ }^{-1}$.
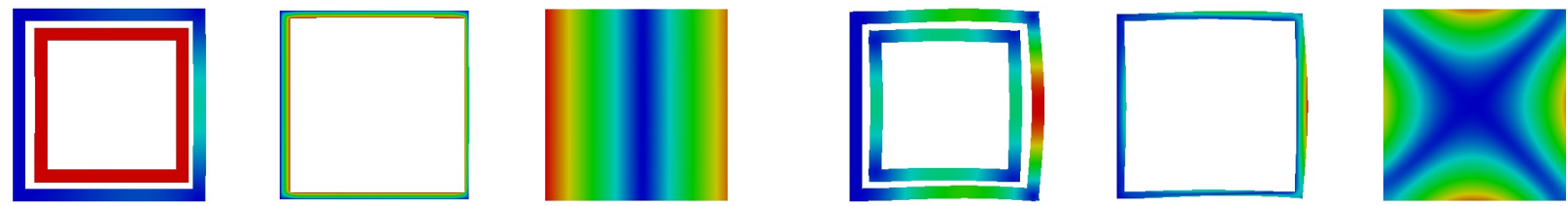

(c) $\eta_{c}=1,5$ - Damped mode 2 (coupled) $-\Omega_{a}=263.48$ rad.s $^{-1}$

Figure 5: Hysteretic model - Cavity filled with water - Visualisation of damped modes 2 and 7 with different hysteretic damping factor $\eta_{c}$. 

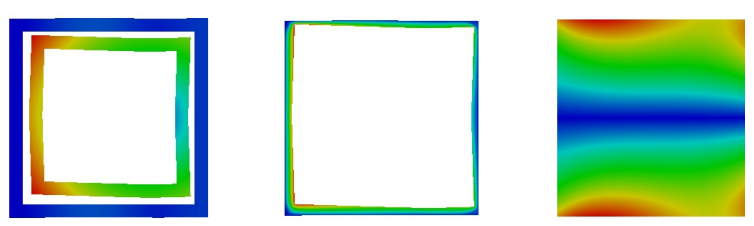

(a) $T=20^{\circ} \mathrm{C}$ - Damped mode 2 (solid) $-\Omega_{a}=605.97$ rad.s ${ }^{-1}$.
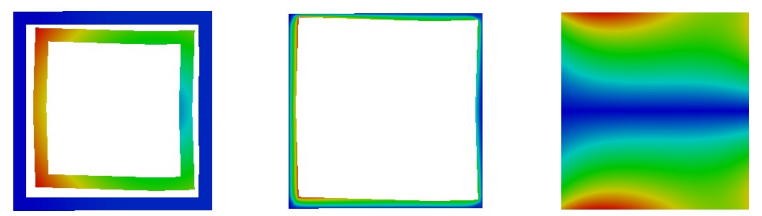

(c) $T=70^{\circ} \mathrm{C}$ - Damped mode 2 (solid) $-\Omega_{a}=340.27$ rad. $\mathrm{s}^{-1}$
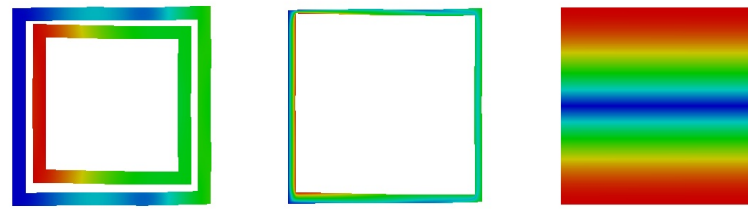

(b) $T=20^{\circ} \mathrm{C}-$ Damped mode 7 (fluid) $-\Omega_{a}=1068.60$ rad.s ${ }^{-1}$
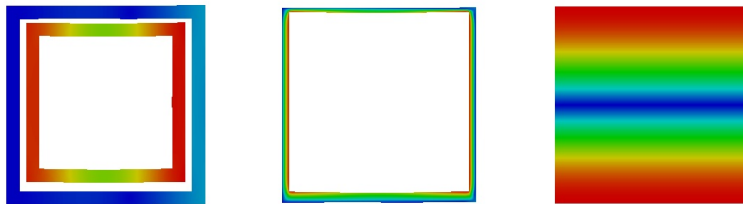

(d) $T=70^{\circ} \mathrm{C}-$ Damped mode 7 (fluid) $-\Omega_{a}=1068.66 \mathrm{rad} . \mathrm{s}^{-1}$

Figure 6: Generalized Maxwell model - Cavity filled with air - Visualisation of damped modes 2 and 7 with different temperatures.
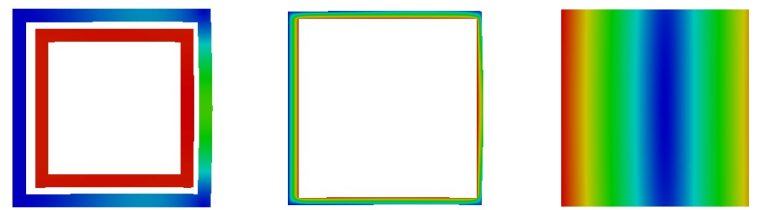

(a) $T=20^{\circ} \mathrm{C}-$ Damped mode 2 (coupled) $-\Omega_{a}=452.38$ rad.s ${ }^{-1}$.
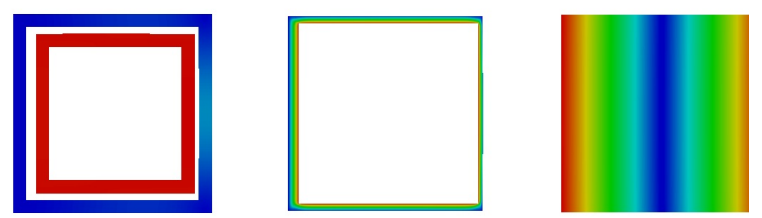

(c) $T=70^{\circ} \mathrm{C}$ - Damped mode 2 (coupled) $-\Omega_{a}=262.75 \mathrm{rad} . \mathrm{s}^{-1}$.
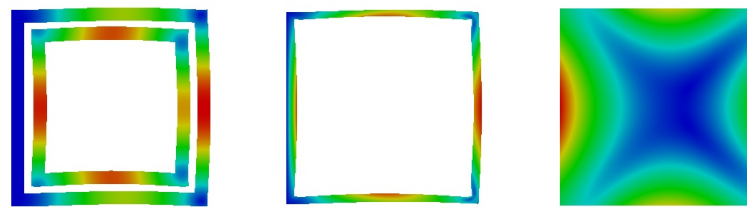

(b) $T=20^{\circ} \mathrm{C}-$ Damped mode 7 (coupled) $-\Omega_{a}=1389.22$ rad.s ${ }^{-1}$.
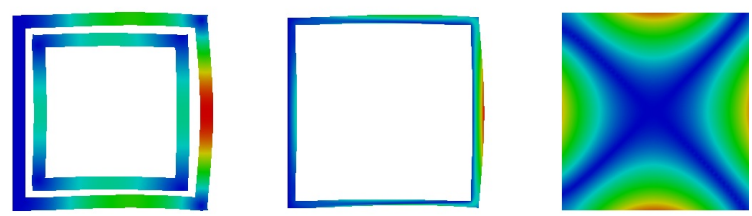

(d) $T=70^{\circ} \mathrm{C}$ - Damped mode 7 (coupled) $-\Omega_{a}=1090.59$ rad.s ${ }^{-1}$.

Figure 7: Generalized Maxwell model - Cavity filled with water - Visualisation of damped modes 2 and 7 with different temperatures. 
(a) Damped angular frequency $\Omega_{a}\left(\right.$ rad.s $\left.^{-1}\right)$

\begin{tabular}{cccccc}
\hline Mode & $\eta_{c}=0.1$ & $\eta_{c}=0.2$ & $\eta_{c}=0.5$ & $\eta_{c}=1.0$ & $\eta_{c}=1.5$ \\
\hline $1(\mathrm{~S})$ & 200.86 & 201.33 & 204.15 & 210.43 & 216.41 \\
$2(\mathrm{~S})$ & 323.27 & 323.31 & 323.61 & 324.67 & 326.43 \\
$3(\mathrm{~S})$ & 336.61 & 336.84 & 338.47 & 344.21 & 353.48 \\
$4(\mathrm{~S})$ & 460.50 & 460.54 & 461.06 & 464.43 & 471.66 \\
$5(\mathrm{~S})$ & 793.12 & 793.08 & 792.79 & 791.45 & 789.02 \\
$6(\mathrm{~S})$ & 1014.23 & 1014.54 & 1016.69 & 1023.89 & 1034.93 \\
$7(\mathrm{~F})$ & 1068.65 & 1068.65 & 1068.65 & 1068.64 & 1068.64 \\
$8(\mathrm{~F})$ & 1068.66 & 1068.66 & 1068.65 & 1068.63 & 1068.62 \\
$9(\mathrm{~S})$ & 1278.00 & 1277.85 & 1276.60 & 1271.30 & 1262.10 \\
$10(\mathrm{~F})$ & 1511.21 & 1511.21 & 1511.21 & 1511.21 & 1511.21 \\
\hline
\end{tabular}

(b) Structural damping $\eta_{m}$

\begin{tabular}{cccccc}
\hline Mode & $\eta_{c}=0.1$ & $\eta_{c}=0.2$ & $\eta_{c}=0.5$ & $\eta_{c}=1.0$ & $\eta_{c}=1.5$ \\
\hline $1(\mathrm{~S})$ & $3.2810^{-2}$ & $6.4610^{-2}$ & $1.4710^{-1}$ & $2.3510^{-1}$ & $2.9110^{-1}$ \\
$2(\mathrm{~S})$ & $9.8810^{-2}$ & $1.9810^{-1}$ & $4.9310^{-1}$ & $9.8010^{-1}$ & 1.45 \\
$3(\mathrm{~S})$ & $9.5110^{-2}$ & $1.9010^{-1}$ & $4.7010^{-1}$ & $9.0810^{-1}$ & 1.29 \\
$4(\mathrm{~S})$ & $8.0610^{-2}$ & $1.6110^{-1}$ & $4.0510^{-1}$ & $8.0710^{-1}$ & 1.19 \\
$5(\mathrm{~S})$ & $1.7010^{-2}$ & $3.4010^{-2}$ & $8.4510^{-2}$ & $1.6810^{-1}$ & $2.5210^{-1}$ \\
$6(\mathrm{~S})$ & $1.1010^{-2}$ & $2.1810^{-2}$ & $5.4010^{-2}$ & $1.0310^{-1}$ & $1.4210^{-1}$ \\
$7(\mathrm{~F})$ & $8.9010^{-6}$ & $1.7710^{-5}$ & $4.3310^{-5}$ & $8.2710^{-5}$ & $1.1910^{-4}$ \\
$8(\mathrm{~F})$ & $1.4810^{-5}$ & $2.9010^{-5}$ & $6.5010^{-5}$ & $9.9010^{-5}$ & $1.1310^{-4}$ \\
$9(\mathrm{~S})$ & $1.4410^{-2}$ & $2.8810^{-2}$ & $7.2010^{-2}$ & $1.4610^{-1}$ & $2.2710^{-1}$ \\
$10(\mathrm{~F})$ & $6.2210^{-6}$ & $1.1610^{-5}$ & $2.2810^{-5}$ & $3.6210^{-5}$ & $4.9710^{-5}$ \\
\hline
\end{tabular}

Table 10: Hysteretic model - Cavity filled with air - Damped angular frequency $\Omega_{a}$ and structural damping $\eta_{m}$ depending on hysteretic damping factor $\eta_{c}$. Indication (F) marks fluid modes and (S) marks solid modes. 
(a) Damped angular frequency $\Omega_{a}\left(\operatorname{rad} . \mathrm{s}^{-1}\right)$

\begin{tabular}{cccccc}
\hline Mode & $\eta_{c}=0,1$ & $\eta_{c}=0,2$ & $\eta_{c}=0,5$ & $\eta_{c}=1,0$ & $\eta_{c}=1,5$ \\
\hline $1(\mathrm{C})$ & 171.06 & 171.57 & 174.70 & 182.12 & 189.13 \\
$2(\mathrm{C})$ & 252.37 & 252.53 & 253.61 & 257.40 & 263.48 \\
$3(\mathrm{C})$ & 300.73 & 300.81 & 301.40 & 303.33 & 306.32 \\
$4(\mathrm{C})$ & 424.06 & 424.10 & 424.55 & 427.54 & 434.14 \\
$5(\mathrm{C})$ & 653.47 & 653.36 & 652.57 & 649.74 & 645.26 \\
$6(\mathrm{C})$ & 933.83 & 933.87 & 934.13 & 934.56 & 934.70 \\
$7(\mathrm{C})$ & 1061.65 & 1061.64 & 1061.59 & 1061.38 & 1061.10 \\
$8(\mathrm{C})$ & 1591.35 & 1592.39 & 1595.88 & 1599.16 & 1601.10 \\
$9(\mathrm{C})$ & 1741.11 & 1741.74 & 1743.68 & 1745.98 & 1747.31 \\
$10(\mathrm{C})$ & 1906.33 & 1953.17 & 1990.79 & 1993.60 & 1994.28 \\
\hline
\end{tabular}

(b) Structural damping $\eta_{m}$

\begin{tabular}{cccccc}
\hline Mode & $\eta_{c}=0,1$ & $\eta_{c}=0,2$ & $\eta_{c}=0,5$ & $\eta_{c}=1,0$ & $\eta_{c}=1,5$ \\
\hline $1(\mathrm{C})$ & $4.1910^{-2}$ & $8.2410^{-2}$ & $1.8710^{-1}$ & $2.9210^{-1}$ & $3.4810^{-1}$ \\
$2(\mathrm{C})$ & $9.5510^{-2}$ & $1.9110^{-1}$ & $4.7310^{-1}$ & $9.1610^{-1}$ & 1.31 \\
$3(\mathrm{C})$ & $9.6710^{-2}$ & $1.9310^{-1}$ & $4.8110^{-1}$ & $9.4910^{-1}$ & 1.40 \\
$4(\mathrm{C})$ & $7.2710^{-2}$ & $1.4610^{-1}$ & $3.6610^{-1}$ & $7.3510^{-1}$ & 1.09 \\
$5(\mathrm{C})$ & $1.7710^{-2}$ & $3.5410^{-2}$ & $8.8510^{-2}$ & $1.7610^{-1}$ & $2.6310^{-1}$ \\
$6(\mathrm{C})$ & $3.5710^{-3}$ & $7.1010^{-3}$ & $1.7510^{-2}$ & $3.3710^{-2}$ & $4.9210^{-2}$ \\
$7(\mathrm{C})$ & $2.1210^{-2}$ & $4.2410^{-2}$ & $1.0610^{-1}$ & $2.1210^{-1}$ & $3.1810^{-1}$ \\
$8(\mathrm{C})$ & $7.9110^{-3}$ & $1.5210^{-2}$ & $3.3410^{-2}$ & $5.9710^{-2}$ & $8.6010^{-2}$ \\
$9(\mathrm{C})$ & $6.8310^{-3}$ & $1.3310^{-2}$ & $3.0810^{-2}$ & $5.7710^{-2}$ & $8.4210^{-2}$ \\
$10(\mathrm{C})$ & $4.9510^{-2}$ & $8.1610^{-2}$ & $3.8510^{-2}$ & $3.7110^{-2}$ & $4.4010^{-2}$ \\
\hline
\end{tabular}

Table 11: Hysteretic model - Cavity filled with water - Damped angular frequency $\Omega_{a}$ and structural damping $\eta_{m}$ depending on hysteretic damping factor $\eta_{c}$. Indication (C) marks coupled modes. 
(a) Damped angular frequency $\Omega_{a}\left(\operatorname{rad} . \mathrm{s}^{-1}\right)$

\begin{tabular}{ccccccc}
\hline Mode & $20^{\circ} \mathrm{C}$ & $30^{\circ} \mathrm{C}$ & $40^{\circ} \mathrm{C}$ & $50^{\circ} \mathrm{C}$ & $60^{\circ} \mathrm{C}$ & $70^{\circ} \mathrm{C}$ \\
\hline $1(\mathrm{~S})$ & 238.48 & 218.54 & 213.72 & 210.13 & 205.80 & 203.15 \\
$2(\mathrm{~S})$ & 605.97 & 428.87 & 398.89 & 381.92 & 358.33 & 340.27 \\
$3(\mathrm{~S})$ & 677.07 & 444.81 & 411.97 & 395.40 & 372.56 & 354.25 \\
$4(\mathrm{~S})$ & 875.21 & 603.27 & 554.77 & 535.32 & 511.04 & 486.98 \\
$5(\mathrm{~S})$ & 906.58 & 860.49 & 834.28 & 825.03 & 816.82 & 807.52 \\
$6(\mathrm{~S})$ & 1141.68 & 1074.36 & 1047.75 & 1039.75 & 1034.36 & 1027.80 \\
$7(\mathrm{~F})$ & 1068.60 & 1068.70 & 1068.68 & 1068.67 & 1068.67 & 1068.66 \\
$8(\mathrm{~F})$ & 1068.64 & 1068.61 & 1068.70 & 1068.71 & 1068.69 & 1068.68 \\
$9(\mathrm{~S})$ & 1161.80 & 1394.33 & 1342.40 & 1326.30 & 1316.30 & 1304.49 \\
$10(\mathrm{~F})$ & 1511.26 & 1511.25 & 1511.23 & 1511.22 & 1511.22 & 1511.22 \\
\hline
\end{tabular}

(b) Structural damping $\eta_{m}$

\begin{tabular}{ccccccc}
\hline Mode & $20^{\circ} \mathrm{C}$ & $30^{\circ} \mathrm{C}$ & $40^{\circ} \mathrm{C}$ & $50^{\circ} \mathrm{C}$ & $60^{\circ} \mathrm{C}$ & $70^{\circ} \mathrm{C}$ \\
\hline $1(\mathrm{~S})$ & $1.7810^{-1}$ & $8.2010^{-2}$ & $4.4110^{-2}$ & $4.8710^{-2}$ & $4.7010^{-2}$ & $3.4610^{-2}$ \\
$2(\mathrm{~S})$ & 1.21 & $4.4310^{-1}$ & $1.7510^{-1}$ & $1.5110^{-1}$ & $1.6910^{-1}$ & $1.3410^{-1}$ \\
$3(\mathrm{~S})$ & $7.6610^{-1}$ & $4.0810^{-1}$ & $1.6610^{-1}$ & $1.4210^{-1}$ & $1.6110^{-1}$ & $1.3010^{-1}$ \\
$4(\mathrm{~S})$ & $7.3510^{-1}$ & $4.4210^{-1}$ & $1.7210^{-1}$ & $1.2410^{-1}$ & $1.4110^{-1}$ & $1.2510^{-1}$ \\
$5(\mathrm{~S})$ & $5.4510^{-1}$ & $1.7710^{-1}$ & $6.1510^{-2}$ & $3.5110^{-2}$ & $3.4210^{-2}$ & $3.2410^{-2}$ \\
$6(\mathrm{~S})$ & $2.3910^{-1}$ & $8.6210^{-2}$ & $3.6310^{-2}$ & $2.0210^{-2}$ & $1.9110^{-2}$ & $1.9910^{-2}$ \\
$7(\mathrm{~F})$ & $5.2610^{-4}$ & $1.4910^{-4}$ & $4.5710^{-5}$ & $2.2710^{-5}$ & $1.9710^{-5}$ & $1.8810^{-5}$ \\
$8(\mathrm{~F})$ & $1.9710^{-4}$ & $1.6910^{-4}$ & $1.6210^{-4}$ & $7.5310^{-5}$ & $5.3710^{-5}$ & $4.1810^{-5}$ \\
$9(\mathrm{~S})$ & $2.6110^{-1}$ & $2.1610^{-1}$ & $7.2610^{-2}$ & $3.5210^{-2}$ & $2.9410^{-2}$ & $2.9810^{-2}$ \\
$10(\mathrm{~F})$ & $2.6810^{-4}$ & $5.9210^{-5}$ & $2.0610^{-5}$ & $1.0210^{-5}$ & $8.3710^{-6}$ & $8.9010^{-6}$ \\
\hline
\end{tabular}

Table 12: Generalized Maxwell model - Cavity filled with air - Damped angular frequency $\Omega_{a}$ and structural damping $\eta_{m}$ depending on the temperature. Indication $(\mathrm{F})$ marks fluid modes and $(\mathrm{S})$ marks solid modes. 
(a) Damped angular frequency $\Omega_{a}\left(\operatorname{rad} . \mathrm{s}^{-1}\right)$

\begin{tabular}{ccccccc}
\hline Mode & $20^{\circ} \mathrm{C}$ & $30^{\circ} \mathrm{C}$ & $40^{\circ} \mathrm{C}$ & $50^{\circ} \mathrm{C}$ & $60^{\circ} \mathrm{C}$ & $70^{\circ} \mathrm{C}$ \\
\hline $1(\mathrm{C})$ & 208.85 & 189.39 & 184.61 & 180.51 & 175.84 & 173.17 \\
$2(\mathrm{C})$ & 452.38 & 327.43 & 306.80 & 293.03 & 274.49 & 262.75 \\
$3(\mathrm{C})$ & 479.74 & 395.00 & 368.72 & 352.93 & 331.07 & 315.30 \\
$4(\mathrm{C})$ & 689.71 & 544.29 & 501.81 & 485.26 & 464.15 & 444.67 \\
$5(\mathrm{C})$ & 726.47 & 705.98 & 687.96 & 680.30 & 672.44 & 664.29 \\
$6(\mathrm{C})$ & 982.85 & 952.01 & 944.19 & 941.75 & 939.89 & 937.73 \\
$7(\mathrm{C})$ & 1389.22 & 1190.69 & 1134.38 & 1117.15 & 1105.13 & 1090.59 \\
$8(\mathrm{C})$ & 1822.20 & 1663.89 & 1629.85 & 1619.74 & 1614.64 & 1609.02 \\
$9(\mathrm{C})$ & 1998.71 & 1818.99 & 1781.32 & 1770.23 & 1764.91 & 1759.23 \\
$10(\mathrm{C})$ & 2100.63 & 2028.92 & 2003.44 & 1990.39 & 1983.39 & 1977.07 \\
\hline
\end{tabular}

(b) Structural damping $\eta_{m}$

\begin{tabular}{ccccccc}
\hline Mode & $20^{\circ} \mathrm{C}$ & $30^{\circ} \mathrm{C}$ & $40^{\circ} \mathrm{C}$ & $50^{\circ} \mathrm{C}$ & $60^{\circ} \mathrm{C}$ & $70^{\circ} \mathrm{C}$ \\
\hline $1(\mathrm{C})$ & $1.9610^{-1}$ & $9.2910^{-2}$ & $5.3510^{-2}$ & $6.2010^{-2}$ & $5.7410^{-2}$ & $4.1210^{-2}$ \\
$2(\mathrm{C})$ & $8.8810^{-1}$ & $3.5210^{-1}$ & $1.5210^{-1}$ & $1.4910^{-1}$ & $1.5710^{-1}$ & $1.1710^{-1}$ \\
$3(\mathrm{C})$ & 1.94 & $4.0910^{-1}$ & $1.6610^{-1}$ & $1.4910^{-1}$ & $1.6510^{-1}$ & $1.2810^{-1}$ \\
$4(\mathrm{C})$ & $6.4410^{-1}$ & $3.7710^{-1}$ & $1.5010^{-1}$ & $1.1510^{-1}$ & $1.2910^{-1}$ & $1.1010^{-1}$ \\
$5(\mathrm{C})$ & $5.1610^{-1}$ & $1.7910^{-1}$ & $6.0610^{-2}$ & $3.7010^{-2}$ & $3.6910^{-2}$ & $3.2810^{-2}$ \\
$6(\mathrm{C})$ & $1.4510^{-1}$ & $3.8210^{-2}$ & $1.3610^{-2}$ & $7.4410^{-3}$ & $6.9910^{-3}$ & $6.8410^{-3}$ \\
$7(\mathrm{C})$ & $8.6010^{-1}$ & $2.4110^{-1}$ & $8.6710^{-2}$ & $4.5010^{-2}$ & $4.0710^{-2}$ & $4.1510^{-2}$ \\
$8(\mathrm{C})$ & $2.0610^{-1}$ & $8.2110^{-2}$ & $3.1410^{-2}$ & $1.5410^{-2}$ & $1.2310^{-2}$ & $1.2910^{-2}$ \\
$9(\mathrm{C})$ & $2.6010^{-1}$ & $8.7810^{-2}$ & $3.2610^{-2}$ & $1.5510^{-2}$ & $1.1810^{-2}$ & $1.2110^{-2}$ \\
$10(\mathrm{C})$ & $1.5610^{-1}$ & $4.6610^{-2}$ & $2.2510^{-2}$ & $1.4910^{-2}$ & $1.3510^{-2}$ & $1.7310^{-2}$ \\
\hline
\end{tabular}

Table 13: Generalized Maxwell model - Cavity filled with water - Damped angular frequency $\Omega_{a}$ and structural damping $\eta_{m}$ depending on the temperature. Indication $(\mathrm{C})$ marks coupled modes. 

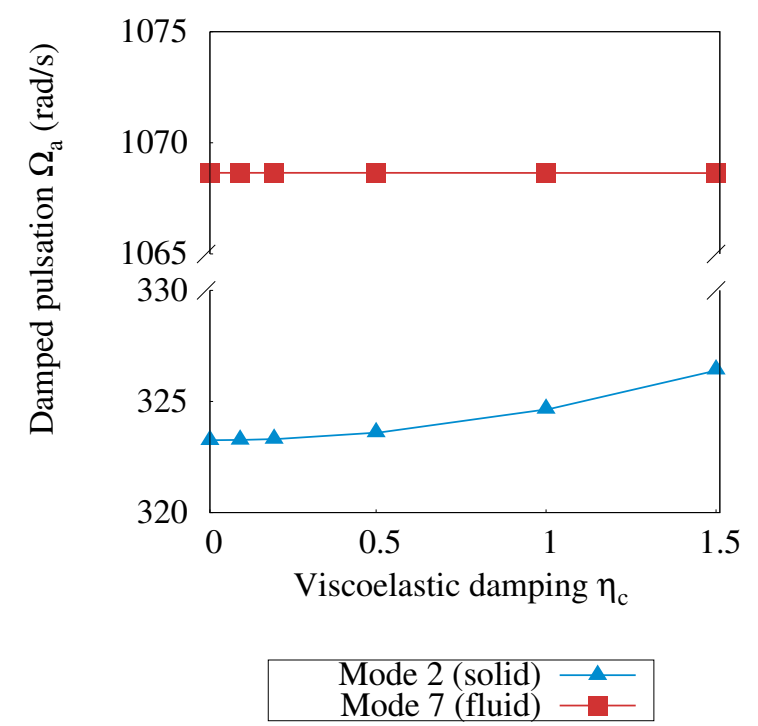

(a) Damped angular frequency $\Omega_{a}$.

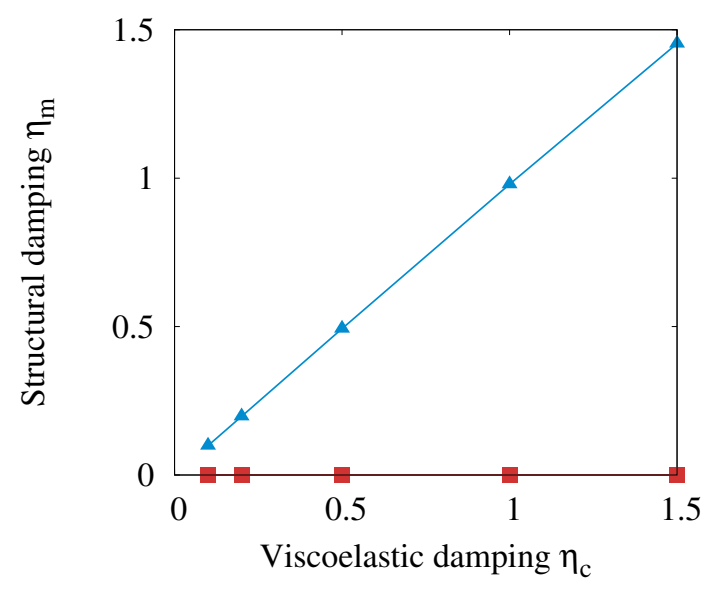

\begin{tabular}{l} 
Mode 2 (solid) $\square-$ \\
Mode 7 (fluid) \\
\hline
\end{tabular}

(b) Structural damping $\eta_{m}$.

Figure 8: Hysteretic model - Cavity filled with air - Evolution of the damped angular frequency $\Omega_{a}$ and the structural damping $\eta_{m}$ depending on hysteretic damping factor $\eta_{c}$.

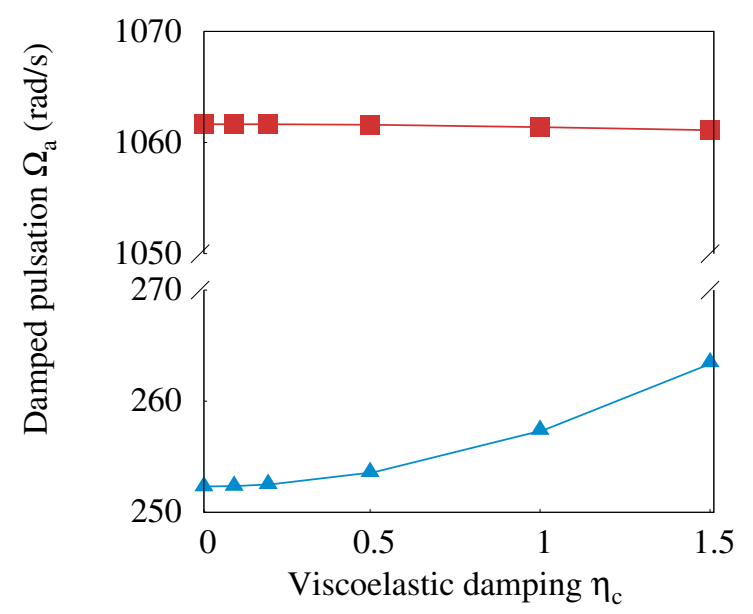

\section{Mode 2 (coupled) Mode 7 (coupled)}

(a) Damped angular frequency $\Omega_{a}$.

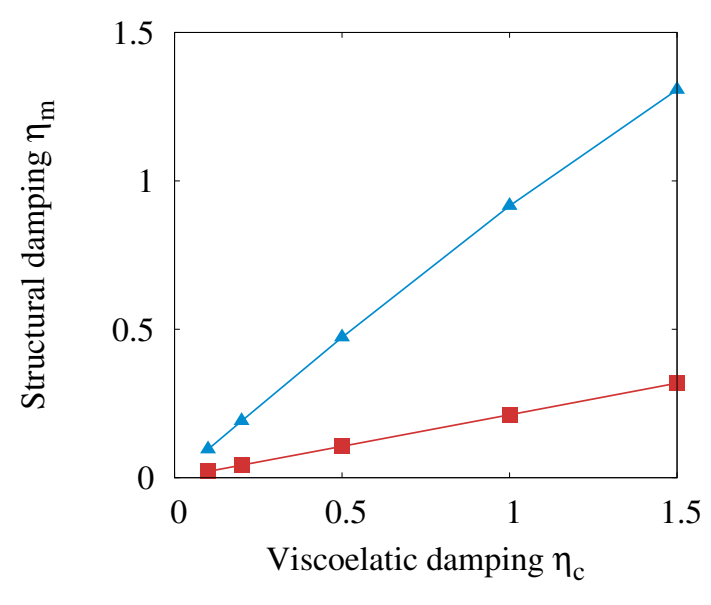

Mode 2 (coupled) Mode 7 (coupled)

(b) Structural damping $\eta_{m}$

Figure 9: Hysteretic model - Cavity filled with water - Evolution of the damped angular frequency $\Omega_{a}$ and the structural damping $\eta_{m}$ depending on hysteretic damping factor $\eta_{c}$. 

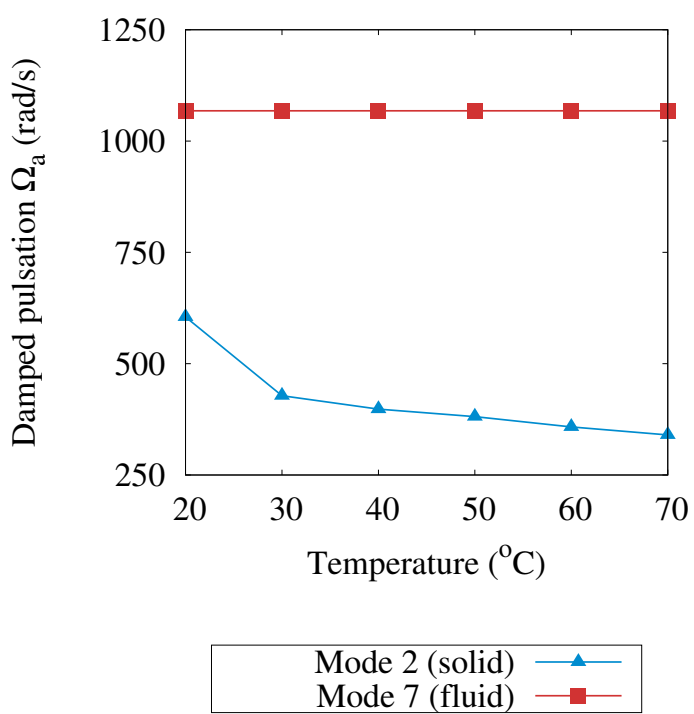

(a) Damped angular frequency $\Omega_{a}$.
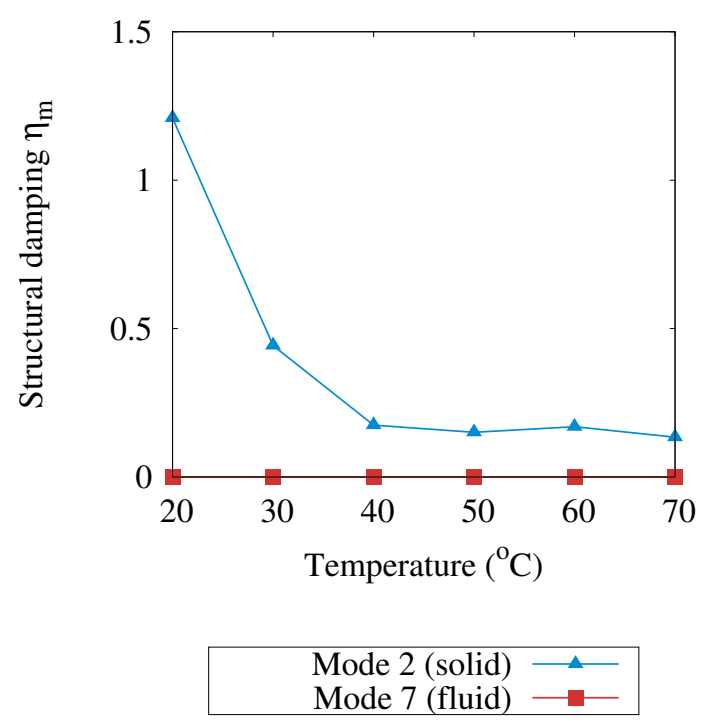

(b) Structural damping $\eta_{m}$.

Figure 10: Generalized Maxwell model - Cavity filled with air - Evolution of the damped angular frequency $\Omega_{a}$ and the structural damping $\eta_{m}$ depending on the temperature for modes 2 and 7 .

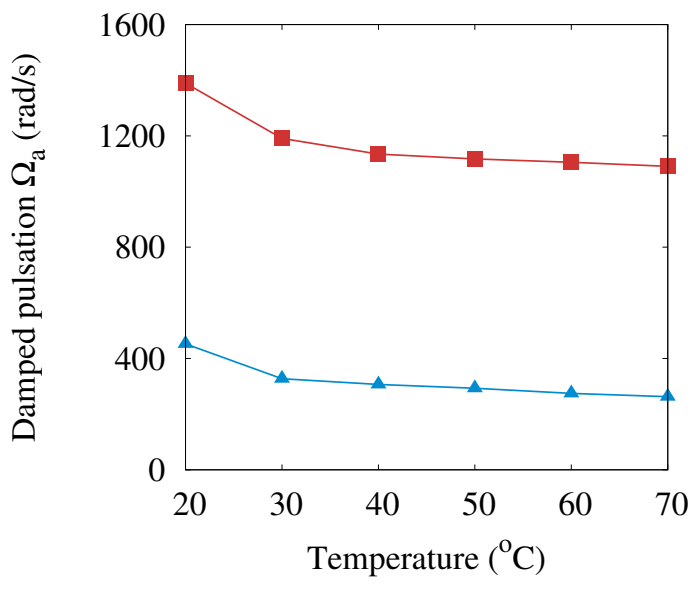

Mode 7 (coupled)

(a) Damped angular frequency $\Omega_{a}$.
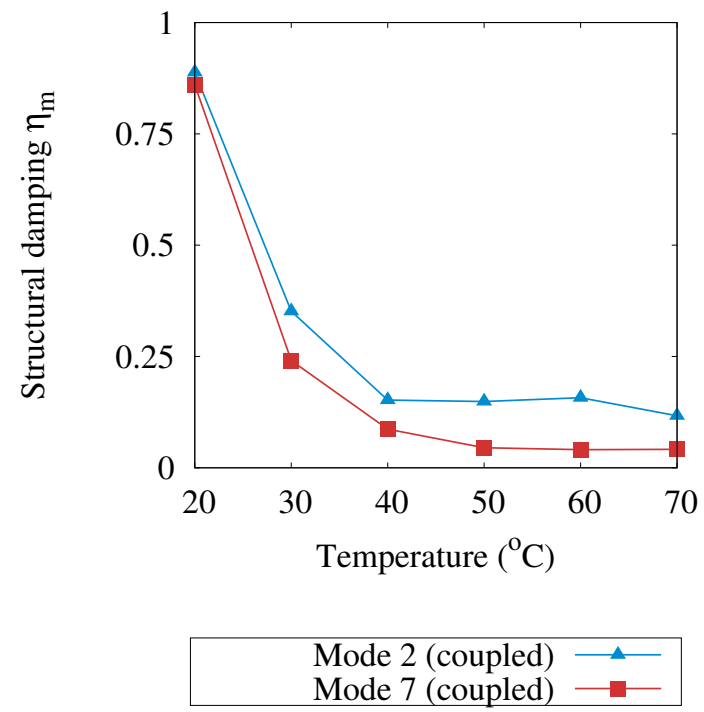

(b) Structural damping $\eta_{m}$.

Figure 11: Generalized Maxwell model - Cavity filled with water - Evolution of the damped angular frequency $\Omega_{a}$ and the structural damping $\eta_{m}$ depending on the temperature for modes 2 and 7.

\section{Conclusion}

In this paper, a High Order Newton solver has been presented to study damped vibrations of a vibroacoustic interior problem. This damping is introduced via a viscoelastic material, included in a sandwich 
structure. Viscoelasticy is modelized by means of a complex Young's modulus. This latter can be constant or dependent of the angular frequency and the temperature. So, the problem to be solved is strongly non-linear.

The proposed solver is based on homotopy and perturbation techniques, according to the method introduced by [15]. First, the HON solver has been validated by comparison with results obtained with the classical Newton algorithm, considered as the reference. Then, performances of the HON solver have been evaluated by comparison with those of the Newton classical method. It appears that the HON solver requires fewer iterations than the classical Newton method. Indeed, the same tangent operator is used to compute a high order polynomial perturbation. Thus, the tangent operator is computed only once per iteration and is conserved for all the orders, contributing to reducing the iterations number. Moreover, the use of Padé representation greatly improves convergence.

Finally, the vibrational behavior of the damped coupled problem has been studied. It appears that the behavior depends on the type of mode considered. For solid and coupled modes, when damping increases, damped angular frequencies and structural damping increase too, whereas for fluid modes, damping does not affect the problem so much.

This work opens onto numerous and various perspectives. Indeed, in this paper, the problem geometry was very simple but has allowed to highlight the relevance of the HON solver. More realistic cases of application can now be studied. For example, a parametric study about position and thickness of the viscoelastic layer is currently in progress. Extension to three-dimensional and external fluid problems are also interesting perspectives. Lastly, recent research-works about bio-based flax composite conducted by Mahmoudi et. al [1] may lead to a study of other viscoelastic constitutive laws.

[1] S. Mahmoudi, A. Kervoelen, G. Robin, L. Duigou, E. M. Daya, J. M. Cadou, Experimental and numerical investigation of the damping of flax-epoxy composite plates, Composite Struct. 208 (15) (2019) 426-433. https://doi.org/10.1016/j.compstruct.2018.10.030.

[2] B. Garnier, Contrôle actif des vibrations [Active control of vibrations], Tech. rep., Techniques de l'ingénieur - Vibrations en milieu industriel, mesures, surveillance et contrôle [Engineering Techniques - Industrial Vibrations, Measurement, Monitoring and Control] (2002).

[3] Livre Blanc de la Recherche en Mécanique - Enjeux industriels et sociétaux. Recherche, innovation, formation. [White paper on mechanical research - industrial and societal challenges. Research, innovation, training.], Tech. rep., Association Française de Mécanique [French Mechanical Association] (2015).

[4] A. Bobillot, E. Balmes, Iterative Techniques for Eigenvalue Solutions of Damped Structures Coupled with Fluids, 43rd AIAA/ASME/ASCE/AHS/ASC Structures, Structural Dynamics, and Materials Conference, American Institute of Aeronautics and Astronautics, Reston, Virigina, 2002. https://doi.org/10.2514/6.2002-1391.

[5] K. Schreiber, Nonlinear Eigenvalue Problems: Newton-type methods and nonlinear Rayleigh functionals, Ph.D. thesis, Technischen Universität Berlin (2008).

[6] M. Hamdaoui, K. Akoussan, E. M. Daya, Comparison of non-linear eigensolvers for modal analysis of frequency dependent laminated visco-elastic sandwich plates, Finite Elements in Analysis and Design. 121 (2016) 75-85. https://doi.org/10.1016/j.finel.2016.08.001.

[7] F. Boumediene, J. M. Cadou, L. Duigou, E. M. Daya, A reduction model for eigensolutions of damped viscoelastic sandwich structures, Mech. Res. Commun. 57 (2014) 74-81. https://doi.org/10.1016/j.mechrescom.2014.03.001.

[8] F. Abdoun, L. Azrar, E. M. Daya, M. Potier-Ferry, Forced harmonic response of viscoelastic structures by an asymptotic numerical method, Comput. Struct. 87 (1-2) (2009) 91-100. https://doi.org/10.1016/j.compstruc.2008.08.006.

[9] G. Dhatt, G. Touzot, Une présentation de la méthode des éléments finis [A presentation of the finite element method], Presses de l'Université Laval Québec - Paris Maloine, 1984.

[10] R. Rikards, A. Chate, E. Barkanov, Finite element analysis of damping the vibrations of laminated composites, Comput. Struct. 47 (6) (1993) 1005-1015. https://doi.org/10.1016/0045-7949(93)90305-W.

295 [11] E. M. Daya, M. Potier-Ferry, A shell finite element for viscoelastically damped sandwich structures, Revue Européenne des Éléments Finis. 11 (1) (2002) 39-56. https://doi.org/10.3166/reef.11.39-56.

[12] C. M. A. Vasques, R. A. S. Moreira, J. Dias Rodrigues, Viscoelastic Damping Technologies-Part I: Modeling and Finite Element Implementation., J. Adv. Res. Mech. Eng. 1 (2) (2010) 76-95.

[13] H. Voss, An Arnoldi Method for Nonlinear Eigenvalue Problems, BIT Numer. Math. 44 (2) (2004) $387-401$. https://doi.org/10.1023/B:BITN.0000039424.56697.8b.

[14] H. Voss, A Jacobi-Davidson method for nonlinear and nonsymmetric eigenproblems, Comput. Struct. 85 (17-18) (2007) 1284-1292. https://doi.org/10.1016/j.compstruc.2006.08.088.

[15] E. Mallil, H. Lahmam, N. Damil, M. Potier-Ferry, An iterative process based on homotopy and perturbation techniques, Comput. Methods Appl. Mech. Eng. 190 (13) (2000) 1845-1858. https://doi.org/10.1016/S0045-7825(00)00198-5.

[16] E. M. Daya, M. Potier-Ferry, A numerical method for nonlinear eigenvalue problems application to vibrations of viscoelastic structures, Comput. Struct. 79 (5) (2001) 533-541. https://doi.org/10.1016/S0045-7949(00)00151-6.

[17] L. Duigou, E. M. Daya, M. Potier-Ferry, Iterative algorithms for non-linear eigenvalue problems. Application to vibrations 
of viscoelastic shells, Comput. Methods Appl. Mech. Eng. 192 (11-12) (2003) 1323-1335. https://doi.org/10.1016/S00457825(02)00641-2.

[18] H. J.-P. Morand, R. Ohayon, Fluid-structure interaction, Wiley, 1995

[19] O. C. Zienkiewicz, R. L. R. L. Taylor, J. Z. Zhu, Finite element method : its basis and fundamentals, ButterworthHeinemann, 2013.

[20] K.-J. Bathe, Finite element procedures, Prentice Hall, 2006.

[21] M. Maess, L. Gaul, Substructuring and model reduction of pipe components interacting with acoustic fluids, Mech. Syst. Signal Process. 20 (1) (2006) 45-64. https://doi.org/10.1016/j.ymssp.2005.02.008.

[22] A. Quarteroni, R. Cacco, F. Saleri, Méthodes Numériques - Algorithmes, analyse et applications [Numerical methods Algorithms, analysis and applications], Springer Milan, Milano, 2007.

[23] P. Deuflhard, Newton Methods for Nonlinear Problems, Springer Berlin Heidelberg, Berlin, Heidelberg, 2011.

[24] B. Claude, L. Duigou, G. Girault, J. M. Cadou, Eigensolutions to a vibroacoustic interior coupled problem with a perturbation method, Comptes Rendus - Mecanique. 345 (2) (2017) 130-136. https://doi.org/10.1016/j.crme.2016.11.002.

[25] B. Claude, L. Duigou, G. Girault, Y. Guevel, J. M. Cadou, Numerical comparison of eigenvalue algorithms for vibroacoustic problems, Mech. Res. Commun. 91 (2018) 39-45. https://doi.org/10.1016/j.mechrescom.2018.05.007.

[26] G. Sliva, A. Brezillon, J. M. Cadou, L. Duigou, A study of the eigenvalue sensitivity by homotopy and perturbation methods, J. Comput. Appl. Math. 234 (7) (2010) 2297-2302. https://doi.org/10.1016/j.cam.2009.08.086.

[27] B. Cochelin, N. Damil, M. Potier-Ferry, Méthode asymptotique numérique, Hermès [Asymptotic Numerical Method], Science publications, 2007.

[28] A. Elhage-Hussein, M. Potier-Ferry, N. Damil, A numerical continuation method based on Padé approximants, Int. J. Solids and Struct. 37 (46-47) (2000) 6981-7001. https://doi.org/10.1016/S0020-7683(99)00323-6.

[29] D. Axelrad, Mechanical models of relaxation phenomena, Adv. Mol. Relax. Process. 2 (1) (1970) 41-68. https://doi.org/10.1016/0001-8716(70)80007-4

[30] L. Rouleau, Modélisation vibro-acoustique de structures sandwich munies de matériaux visco-élastiques. [Vibroacoustic modeling of sandwich structures with viscoelastic materials], Ph.D. thesis, Conservatoire national des arts et métiers CNAM (2013).

[31] J. Landier, Modélisation et étude expérimentale des propriétés amortissantes des tôles sandwich [Modeling and experimental study of the damping properties of sandwich sheets], Ph.D. thesis, Université de Metz (1993).

[32] L. Duigou-Kersulec, Modélisation numérique de l'amortissement passif et actif des tôles sandwich comportant des couches viscoélastiques ou piézoélectriques [Numerical modelling of passive and active damping of sandwich sheets with viscoelastic or piezoelectric layers], Ph.D. thesis, Université de Metz (2002).

[33] A. Bermúdez, R. Rodríguez, Finite element computation of thevibration modes of a fluid-solid system, Comput. Methods Appl. Mech. Eng. 119 (3-4) (1994) 355-370. https://doi.org/10.1016/0045-7825(94)90095-7. 